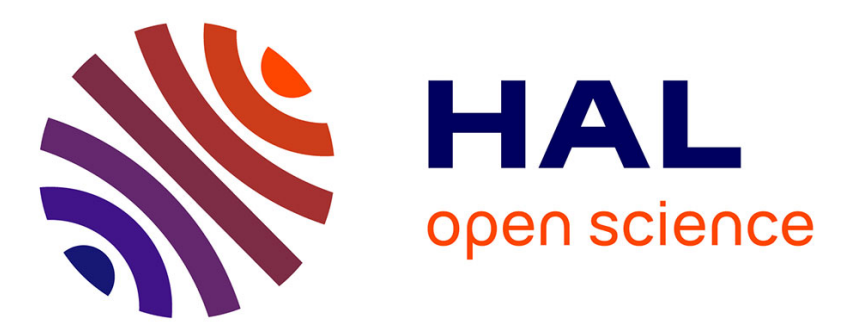

\title{
Les élections présidentielles dans une localité de la région parisienne - notes de recherche
}

Janine Mossuz-Lavau, Guy Michelat

\section{To cite this version:}

Janine Mossuz-Lavau, Guy Michelat. Les élections présidentielles dans une localité de la région parisienne - notes de recherche. Revue Française de Science Politique, 1966, 16 (3), pp.548-580. 10.3406/rfsp.1966.392944 . hal-01000222

HAL Id: hal-01000222

https://hal-sciencespo.archives-ouvertes.fr/hal-01000222

Submitted on 4 Jun 2014

HAL is a multi-disciplinary open access archive for the deposit and dissemination of scientific research documents, whether they are published or not. The documents may come from teaching and research institutions in France or abroad, or from public or private research centers.
L'archive ouverte pluridisciplinaire HAL, est destinée au dépôt et à la diffusion de documents scientifiques de niveau recherche, publiés ou non, émanant des établissements d'enseignement et de recherche français ou étrangers, des laboratoires publics ou privés.

\section{(1) (1) $\$$}

Distributed under a Creative Commons Attribution - NonCommercial - NoDerivatives 44.0 


\title{
NOTES DE RECHERCHE
}

\section{Les Elections Présidentielles dans une Localité de la Région Parisienne}

\author{
GUY MICHELAT et JANINE MOSSUZ
}

La Fondation nationale des sciences politiques, en collaboration avec l'Institut français d'opinion publique, a effectué, au lendemain de l'élection présidentielle, un sondage d'opinion sur la population de BoulogneBillancourt 1. C'est à partir des données de cette enquête qu'a été entreprise cette analyse du vote aux deux tours de scrutin de l'élection prési. dentielle 2 .

On a envisagé successivement les transferts de voix entre le premier et le second tour, les rapports entre les électorats des candidats à la Présidence et certaines variables politiques et sociologiques, puis l'analyse des hésitations qui ont pu précéder les votes, enfin les motivations déclarées de ces votes.

\section{LES ELECTORATS}

\section{Transferts de voix du premier au second tour de scrutin}

Les électeurs du général de Gaulle et de François Mitterrand leur sont dans l'ensemble fidèles. Des électeurs de Jean Lecanuet, $47 \%$ votent de Gaulle au second tour, $32 \%$ Mitterrand et $7 \%$ déclarent ne pas avoir voté; 12 disent avoir voté sans préciser pour qui. Les électeurs

1. Cette enquête a été réalisée gråce au concours de la Délégation générale à la recherche scientifique et technique.

2. Sondage aréolaire, de $142 \%$ interviews, réalisé dans la première moitié du mois de janvier 1966. 


\section{Les Ellections Présidentielles}

de Jean-Louis Tixier-Vignancour reportent surtout leurs voix sur Mitterrand $(63 \%)$, mais $14 \%$ ont voté de Gaulle et $7 \%$ disent qu'ils se sont abstenus.

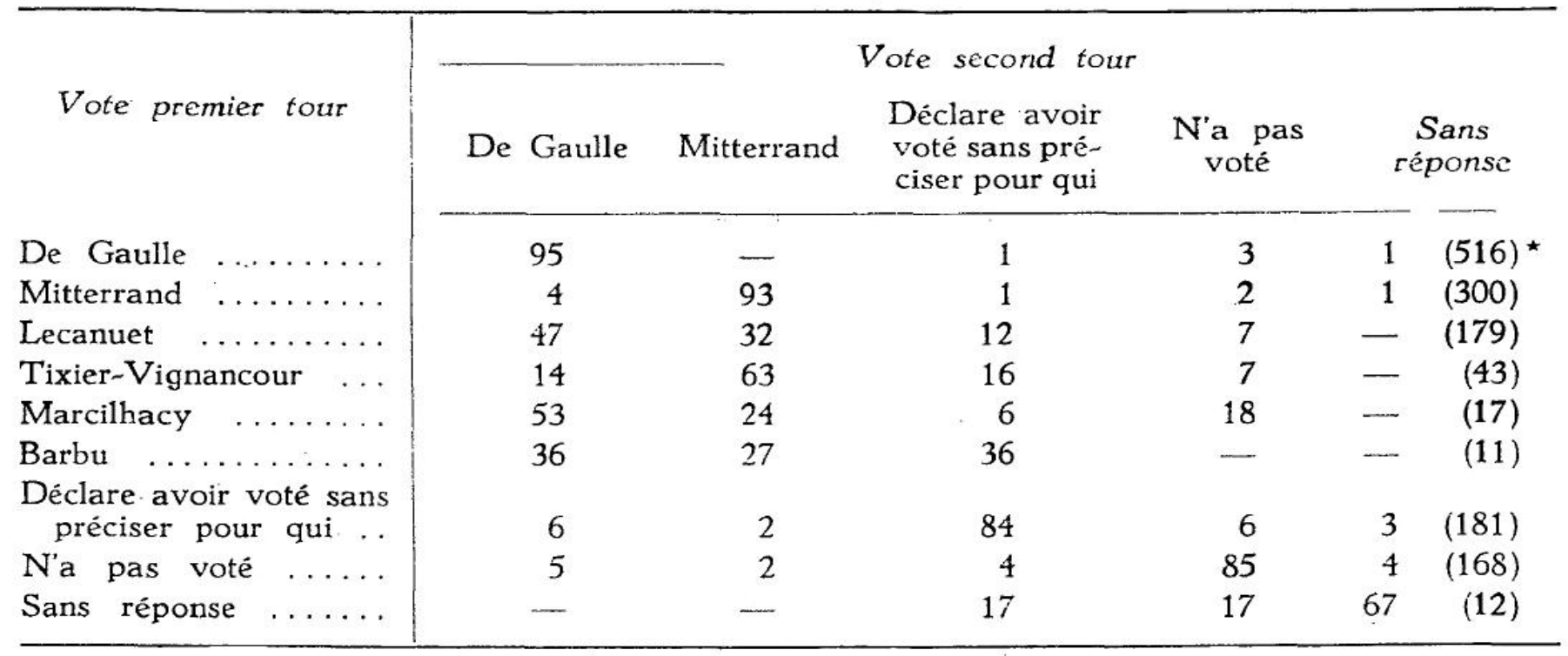

Si on établit les pourcentages par rapport au second tour, on voit que $78 \%$ (626)* de l'électorat du général de Gaulle et $74 \%$ (376) de celui de François Mitterrand est constitué par des électeurs du premier tour. S'y ajoute une proportion semblable d'électeurs de Jean Lecanuet (14\% pour de Gaulle, $15 \%$ pour Mitterrand); $7 \%$ de l'électorat de François Mitterrand au second tour provient de l'électorat de Jean-Louis TixierVignancour.

\section{Electorats des élections présidentielles et prétérences politiques}

Le tableau suivant donne la composition politique de chacun des électorats du premier tour; il est établi à partir de l'ensemble des seules personnes qui ont répondu à la question : "S'il $y$ avait à l'beure actuelle des élections législatives pour choisir des députés, parmi les candidats suivants quel est celui pour lequel il $y$ aurait les plus grandes chances que vous votiez?》

* Tous les résultats donnés au cours de cet article le sont sous forme de pourcentages; le chiffre entre parenthèses indique l'effectif sur lequel est calculé le pourcentage. 


\section{Notes de Recherche}

\begin{tabular}{|c|c|c|c|c|c|}
\hline $\begin{array}{c}\text { Vote } \\
\text { premier tour }\end{array}$ & $\begin{array}{l}\text { « Gauche » (P.C.. } \\
\text { P.S.U., S.F.I.O., } \\
\text { radicaux) }\end{array}$ & $\begin{array}{l}\text { «Centristes » } \\
\text { (C.N.I., M.R.P.) }\end{array}$ & $\begin{array}{l}\text { «Gaullistes » } \\
\text { (UI.N.R.-U.D.T., } \\
\text { Rép. indép.) }\end{array}$ & & $\begin{array}{l}\text { trême- } \\
\text { site » }\end{array}$ \\
\hline $\begin{array}{l}\text { Mitterrand } \\
\text { De Gaulle } \ldots \ldots \ldots \\
\text { Lecanuet ......... } \\
\text { Tixier-Vignancour . }\end{array}$ & $\begin{array}{l}91 \\
15 \\
23 \\
14\end{array}$ & $\begin{array}{r}5 \\
14 \\
55 \\
60\end{array}$ & $\begin{array}{r}4 \\
70 \\
20 \\
6\end{array}$ & $\begin{array}{r}0 \\
2 \\
1 \\
20\end{array}$ & $\begin{array}{r}(248) \\
(381) \\
(139) \\
(35)\end{array}$ \\
\hline
\end{tabular}

On constate une grande coïncidence de l'électorat théorique de François Mitterrand (les partis qui le soutiennent officiellement) et de son électorat réel 3. L'hétérogénéité des autres électorats est plus grande : celui du général de Gaulle, s'il comprend $70,0 \%$ de «gaullistes 》, comprend aussi $15 \%$ d'électeurs «de gauche 》 et $14 \%$ d'électeurs «centristes 》. L'électorat de Jean Lecanuet ne comprend qu'un peu plus de la moitié d'électeurs «centristes», mais il comprend par ailleurs 23 rc d'électeurs « de gauche » et $20 \%$ d'électeurs " gaullistes » 4 . Enfin l'électorat de Jean-Louis Tixier-Vignancour est surtout composé d'électeurs du centre et d'extrêmedroite, mais aussi d'électeurs « de gauche ».

Le second tour augmente l'hétérogénéité des électorats : celui de François Mitterrand dont l'essentiel est composé de voix «de gauche», comprend aussi $171 \%$ ce voix «centristes 》; celui du général de Gaulle comprend le même pourcentage de voix « de gauche » qu'au premier tour, mais aussi une plus grande proportion de voix «centristes 》 $\overline{0}$.

\begin{tabular}{|c|c|c|c|c|c|c|}
\hline $\begin{array}{c}\text { Vote } \\
\text { second tour }\end{array}$ & (P.C., & $\begin{array}{l}\text { «Gauche » } \\
\text { P.S.U., S.F.I.O., } \\
\text { radicaux) }\end{array}$ & $\begin{array}{l}\text { «Centristes » } \\
\text { (C.N.I., M.R.P.) }\end{array}$ & $\begin{array}{l}\text { «Gaullistes » } \\
\text { (U.N.R.-U.D.T., } \\
\text { Rép. indép.) }\end{array}$ & \multicolumn{2}{|c|}{$\begin{array}{l}\text { «xtrême- } \\
\text { droite» }\end{array}$} \\
\hline $\begin{array}{l}\text { Mitterrand } \\
\text { De Gaulle }\end{array}$ & & $\begin{array}{l}79 \\
15\end{array}$ & $\begin{array}{l}17 \\
21\end{array}$ & $\begin{array}{r}3 \\
62\end{array}$ & $\begin{array}{l}1 \\
2\end{array}$ & $\begin{array}{l}(311) \\
(459)\end{array}$ \\
\hline
\end{tabular}

3. En considérant l'ensemble des électeurs de François Mitterrand (300), précisons que cet électorat comprend en particulier $34 \%$ de communistes, $26 \%$ d'électeurs S.F.I.O. $14 \zeta_{i c}$ d'électeurs P.S.U. et $17 \%$ de personnes qui ne se prononcent pas sur leur vote à des élections législatives.

4. Précisons que sur l'ensemble des réponses, l'électorat de Jean Lecanuet est composé de 23 \% d'électeurs du C.N.I., de $20 \%$ d'électeurs du M.R.P., de $12 \%$ d'électeurs de la S.F.I.O. et de $22 \%$ d'électeurs qui ne répondent pas à la question sur d'éventuelles législatives.

5. $\mathrm{Si}$ on fait la ventilation par parti sur l'ensemble des réponses, l'électorat de François Mitterrand est composé de $28 \%$ d'électeurs communistes, de $24 \%$ d'électeurs S.F.I.O., de $11 \%$ d'électeurs P.S.U., mais aussi de $9 \%$ d'électeurs du C.N.I., de $5 \%$ d'électeurs M.R.P. et de $18 \%$ de sans réponse à la question concernant les législatives.

L'électorat du général de Gaulle comprend $38 \%$ de voix U.N.R.-U.D.T., $10 \%$ de voix du C.N.I., $6 \%$ de voix du M.R.P., $7 \%$ de voix de la S.F.I.O., $2 \%$ de voix du P.C. et $27 \%$ de sans réponse. 


\section{Electorat des elections présidentielles et variables sociologiques}

$S E X E$

La proportion des hommes dans l'électorat de François Mitterrand est plus importante que dans l'électorat du général de Gaulle et dans celui de Jean Lecanuet (qui sont d'autre part semblables entre eux), que ce soit au premier ou au second tour de scrutin; c'est un phénomène constant que l'électorat «de gauche » soit plus masculin que féminin 6.

\section{$A G E$}

L'électorat de Jean Lecanuet est celui qui comprend le plus d'électeurs de moins de 43 ans et celui du général de Gaulle est celui qui en comprend le moins. Rappelons qu'en 1962 les électorats les plus «jeunes » étaient ceux du P.C. et du P.S.U., les plus «vieux» étaient ceux de l'U.N.R.-U.D.T. et des radicaux 7.

\section{NIVEAU D'ETUDES}

Le niveau d'études des électeurs de Jean Lecanuet est supérieur à celui des électeurs du général de Gaulle et surtout de François Mitterrand. En 1962 l'électorat «de gauche » suscitait des remarques analogues 8. Entre le premier et le second tour la composition de l'électorat de François Mitterrand tend à se rapprocher de celui du général de Gaulle.

\section{NIVEAU DE REVENUS}

L'électorat de Jean Lecanuet est, de beaucoup, celui où le niveau de revenus est le plus élevé. L'électorat du général de Gaulle et celui de François Mitterrand sont relativement proches l'un de l'autre, mais les électeurs du «candidat de la gauche» sont plus nombreux à avoir

6. Dogan (Mattei), «Les attitudes politiques des femmes en Europe et aux Etats-Unis $\gg$, pp. 283-302 in Boudon (Raymond), Lazarsfeld (Paul), Le vocabulaire des sciences sociales, Paris, La Haye. Mouton, 1965. Michelat (Guy), «Attitudes et comportements politiques à l'automne 1962 », p. 228 in : Le référendum d'octobre et les élections de novembre 1962, sous la direction de François Goguel, Paris, A. Colin, 1965 (Cahiers de la Fondation nationale des sciences politiques, 142 ).

7. Ibid., p. 230.

8. Ibid., p. 232. 


\section{Notes de Recherche}

des revenus inférieurs à $1200 \mathrm{~F}$, comme on a pu aussi le constater en 19629.

Comme pour le niveau d'études on constate qu'au second tour l'électorat de François Mitterrand devient presque semblable à celui du général de Gaulle quant au niveau de revenus. Cela est vraisemblablement dû au report d'une partie des voix de Jean Lecanuet.

\section{PROFESSION DE LA PERSONNE INTERROGEE (10)}

L'électorat de Jean Lecanuet se caractérise par une plus grande proportion de professions libérales et cadres supérieurs et d' «inactifs » et par une moindre proportion d'employés et d'ouvriers; s'y oppose l'électorat du

\begin{tabular}{|c|c|c|c|c|c|}
\hline & \multicolumn{3}{|c|}{ Vote premier tour } & \multicolumn{2}{|c|}{ Vote second tour } \\
\hline & De Gaulle & Mitterrand & Lecanuet & De Gaulle & Mitterrand \\
\hline $\begin{array}{l}\text { Sexe } \\
\quad \text { Hommes } \quad \ldots \ldots \ldots \ldots \ldots \\
\text { Femmes } \quad \ldots \ldots \ldots \ldots \ldots\end{array}$ & $\begin{array}{l}46 \\
54\end{array}$ & $\begin{array}{l}55 \\
45\end{array}$ & $\begin{array}{l}47 \\
53\end{array}$ & $\begin{array}{l}47 \\
53\end{array}$ & $\begin{array}{l}55 \\
45\end{array}$ \\
\hline $\begin{array}{l}\text { Age } \\
\text { Moins de } 43 \text { ans } \ldots \ldots \\
\text { Plus de } 43 \text { ans } \ldots . . .\end{array}$ & $\begin{array}{l}40 \\
58\end{array}$ & $\begin{array}{l}48 \\
48\end{array}$ & $\begin{array}{l}57 \\
40\end{array}$ & $\begin{array}{l}42 \\
55\end{array}$ & $\begin{array}{l}49 \\
48\end{array}$ \\
\hline $\begin{array}{l}\text { Niveau d'études } \\
\text { Pas d'études, primaire } \\
\text { Primaire sup., technique, } \\
\text { com.: } \\
\text { Secondaire } \ldots \ldots \ldots \ldots \ldots \\
\text { Supérieur } \ldots \ldots \ldots \ldots \ldots\end{array}$ & $\begin{array}{l}43 \\
18\end{array}$ & $\begin{array}{l}41 \\
14\end{array}$ & $\begin{array}{l}49 \\
34\end{array}$ & $\begin{array}{l}44 \\
19\end{array}$ & $\begin{array}{l}42 \\
19\end{array}$ \\
\hline $\begin{array}{l}\text { Niveau de revenus } \\
\text { Moins de } 1200 \mathrm{~F} \ldots . \\
\text { Plus de } 1200 \mathrm{~F} \ldots \ldots\end{array}$ & $\begin{array}{l}39 \\
52\end{array}$ & $\begin{array}{l}45 \\
49\end{array}$ & $\begin{array}{l}19 \\
71\end{array}$ & $\begin{array}{l}36 \\
55\end{array}$ & $\begin{array}{l}39 \\
54\end{array}$ \\
\hline $\begin{array}{l}\text { Profession de la personne } \\
\text { interrogée } \\
\text { Patrons ind. et com- } \\
\text { merce } \ldots \ldots \ldots \ldots \\
\text { Prof. lib., cadres sup. } \\
\text { Cadres moyens } \ldots \ldots \ldots \\
\text { Employés } \ldots \ldots \ldots \ldots \ldots \\
\text { Ouvriers } \ldots \ldots \ldots \ldots \\
\text { Pers. service } \ldots \ldots \ldots \\
\text { Autres cat. } \ldots \ldots \ldots \ldots \\
\text { Inactifs } \ldots \ldots \ldots \ldots\end{array}$ & $\begin{array}{r}9 \\
13 \\
15 \\
11 \\
15 \\
7 \\
2 \\
28 \\
(516)\end{array}$ & $\begin{array}{r}7 \\
8 \\
16 \\
13 \\
29 \\
4 \\
2 \\
22 \\
(300)\end{array}$ & $\begin{array}{r}7 \\
19 \\
14 \\
3 \\
11 \\
4 \\
2 \\
40 \\
(179)\end{array}$ & $\begin{array}{r}9 \\
14 \\
15 \\
11 \\
14 \\
6 \\
2 \\
29 \\
(627)\end{array}$ & $\begin{array}{r}7 \\
10 \\
16 \\
11 \\
26 \\
4 \\
2 \\
25 \\
(376)\end{array}$ \\
\hline
\end{tabular}

9. Ibid., p. 234.

10. Les catégories socio-professionnelles utilisées sont celles de l'Institut national de la statistique et des études économiques. 


\section{Les Elections Présidentielles}

«candidat de la gauche » qui comprend le plus d'ouvriers et d'employés et le moins de professions libérales et de cadres supérieurs. Entre ces deux électorats, celui du général de Gaulle occupe une position intermédiaire. D'un tour à l'autre la composition socio-professionnelle de l'électorat gaulliste ne se modifie pratiquement pas, mais celui de François Mitterrand tend à s'en rapprocher; les différences importantes qui subsistent sont dues à la plus faible proportion de professions libérales et cadres supérieurs et à la plus forte proportion d'ouvriers dans l'électorat de François Mitterrand.

\section{LES HESITATIONS AVANT LE VOTE}

\section{Hésitations avant le scrutin et vote effectif}

Au premier tour de l'élection présidentielle, $881 \%$ des électeurs de Mitterrand, $87 \%$ des électeurs du général de Gaulle et $81 \%$ des électeurs de Tixier-Vignancour n'ont pas du tout hésité avant de voter. En revanche, 66.\% seulement des électeurs de Lecanuet n'ont pas hésité. Il semble que son électorat soit le plus indécis, peut-être parce que le candidat du Centre démocrate était peu connu avant la campagne électorale, et sans doute aussi de par sa position entre de Gaulle et Mitterrand. Si l'on considère les personnes qui ont hésité avant de voter pour lui, on s'aperçoit que $531 \%$ d'entre elles ont hésité entre dé Gaulle et Lecanuet et $27 \%$ entre Mitterrand et Lecanuet. Chez les électeurs de François Mitterrand, on peut constater le même attrait pour le général de Gaulle : $361 \%$ de ceux qui ont hésité envisageaient de voter soit pour lui, soit pour le général de Gaulle, alors que $22 \% \%$ hésitaient entre Mitterrand et Lecanuet. Quant aux électeurs qui, après avoir hésité, ont finalement voté pour le général de Gaulle, $49 . \%$ d'entre eux ont hésité entre Lecanuet et de Gaulle, 35:\% entre Mitterrand et de Gaulle. Cette différence semble provenir du fait que les électorats centriste et gaulliste sont plus proches l'un de l'autre; la crainte des communistes a pu déterminer des électeurs désireux d'un changement à envisager de voter pour Lecanuet plutôt que pour Mitterrand. D'ailleurs, si l'on totalise les cas où il y a eu hésitation pour chacun des trois candidats, on s'aperçoit que $58 \%$ des hésitants pensaient - entre autres - à de Gaulle, $541 \%$ à Lecanuet et $37 \%$ à Miterrand. On pourrait donc faire l'hypothèse que du moins parmi les hésitants l'électorat potentiel du général de Gaulle et du candidat centriste a eu à peu près la même importance, mais que celui de François Mitterrand était beaucoup plus restreint. 


\begin{tabular}{|c|c|c|c|c|c|}
\hline \multirow[t]{2}{*}{ Vote premier tour } & $\begin{array}{c}\text { Au premier } \\
\text { beaucoup } \\
\text { hésité? }\end{array}$ & $\begin{array}{l}\text { de l'éle } \\
\text { un peu } \\
\text { hésité? }\end{array}$ & $\begin{array}{l}\text { on présidentie } \\
\text { pas du tout } \\
\text { hésité? }\end{array}$ & $\begin{array}{c}\text { e, est-ce } \\
\text { Sans } \\
\text { réponse }\end{array}$ & vous avez... \\
\hline & & 10 & 87 & & $(516)$ \\
\hline$\ldots \ldots \ldots \ldots$ & 3 & 9 & 88 & & $(300)$ \\
\hline Lecanuet $\ldots . .$. & 10 & 24 & 66 & & $(178)$ \\
\hline Tixier-Vignancour & 12 & & 81 & & (43) \\
\hline $\begin{array}{r}\text { Déclarent avoir voté sans } \\
\text { préciser pour qui } \ldots\end{array}$ & 3 & 14 & 81 & 1 & $(181)$ \\
\hline N'ont pas voté $\ldots \ldots \ldots$ & 2 & 8 & 57 & 34 & $(168)$ \\
\hline Ensemble $\ldots \ldots \ldots \ldots$ & 4 & 12 & 79 & 5 & $(1+27)$ \\
\hline
\end{tabular}

\begin{tabular}{|c|c|c|c|c|c|}
\hline \multirow{2}{*}{$\begin{array}{l}\text { Vote premier tour } \\
\text { Entre quels candidats?... }\end{array}$} & \multicolumn{2}{|c|}{ Parmi le } & \multicolumn{2}{|c|}{$\begin{array}{l}\text { personnes qui ont «beaucout } \\
\text { ou «un peu hésité...» }\end{array}$} & hésité》 \\
\hline & $\mathrm{De}$ & Gaulle & Mitterrand & Lecanuet & Ensemble \\
\hline De Gaulle et Lecanuet...$\ldots$. & & 49 & & & \\
\hline De Gaulle et Mitterrand ...... & & 35 & 36 & & 20 \\
\hline Mitterrand et Lecanuet ........ & & & 22 & 27 & 13 \\
\hline $\begin{array}{l}\text { De Gaulle et } \\
\text { Tixier-Vignancour } \ldots \ldots \ldots \ldots \\
\text { ou Marcilhacy } \ldots \ldots \ldots \ldots \ldots \ldots \\
\text { ou Barbu } \ldots \ldots \ldots \ldots \ldots\end{array}$ & & & & & \\
\hline $\begin{array}{l}\text { Mitterrand et } \\
\text { Tixier }- \text { Vignancour } \ldots \ldots \ldots \ldots \\
\text { ou Marcilhacy } \ldots \ldots \ldots \ldots \ldots \\
\text { ou Barbu } \ldots \ldots \ldots \ldots \ldots\end{array}$ & & & & & \\
\hline $\begin{array}{l}\text { Lecanuet et } \\
\quad \text { Tixier-Vignancour } \quad \ldots \ldots \ldots \ldots \\
\text { ou Marcilhacy } \ldots \ldots \ldots \ldots \ldots \\
\text { ou Barbu } \ldots \ldots \ldots \ldots \ldots\end{array}$ & & & & 12 & \\
\hline $\begin{array}{l}\text { De Gaulle et Mitterrand } \\
\text { et Lecanuet } \ldots \ldots \ldots \ldots \ldots \ldots\end{array}$ & & & & 2 & \\
\hline Autres types d'hésitation ..... & & 3 & 14 & 3 & $?$ \\
\hline Sans réponse $\ldots \ldots \ldots \ldots \ldots \ldots$ & & $\begin{array}{c}7 \\
(67)\end{array}$ & $\begin{array}{c}11 \\
(36)\end{array}$ & $\begin{array}{c}3 \\
(60)\end{array}$ & $\begin{array}{c}13 \\
(231)\end{array}$ \\
\hline
\end{tabular}


Parmi les électeurs du général de Gaulle et de François Mitterrand, fidèles à leur candidat du premier au second tour, la proportion d'hésitants au premier tour est pratiquement la même. On a vu que les électeurs de Lecanuet étaient ceux qui avaient le plus hésité au premier tour; si on sépare ceux qui au second tour ont choisi Mitterrand et ceux qui ont choisi de Gaulle, on voit que les derniers avaient beaucoup plus hésité que les premiers, ce qui semble normal puisque les électeurs de Lecanuet avaient surtout hésité entre de Gaulle et Lecanuet. Mais cela veut aussi dire que la partie de l'électorat centriste qui avait le moins hésité au premier tour, qui avait choisi avec le moins d'hésitations Lecanuet était aussi celle qui avait voté contre de Gaulle; le passage au vote Mitterrind s'est alors fait plus facilement puisqu'il incarnait l'opposition au général de Gaulle.

\begin{tabular}{|c|c|c|c|c|}
\hline Vote $1^{\text {er }}$ tour & Vote $2^{\mathrm{e}}$ tour & Hésitants & Non & hésitants \\
\hline $\begin{array}{l}\text { De Gaulle } \\
\text { Mitterrand } \\
\text { Lecanuet } \\
\text { Lecanuet }\end{array}$ & $\begin{array}{l}\text { De Gaulle } \\
\text { Mitterrand } \\
\text { De Gaulle } \\
\text { Mitterrand }\end{array}$ & $\begin{array}{l}12 \\
11 \\
30 \\
44\end{array}$ & $\begin{array}{l}88 \\
89 \\
70 \\
56\end{array}$ & $\begin{array}{r}(491) \\
(278) \\
(86) \\
(57)\end{array}$ \\
\hline
\end{tabular}

\section{Hésitations avant le scrutin et préférences politiques}

La faiblesse de certains effectifs ne permet pas d'analyser très finement les hésitations des électeurs des trois principaux candidats en fonction de leur vote lors d'éventuelles élections législatives. Toutefois, on peut voir que, parmi les électeurs du général de Gaulle, c'est chez les électeurs U.N.R.-U.D.T. qu'il y a le moins d'hésitants $(8, \mathrm{l}, \mathrm{C})(225)$ alors que le pourcentage d'hésitants est de $19 \%$ (52) pour les électeurs «centristes» et de 21.

Dans l'électorat de François Mitterrand ce sont les communistes qui ont le moins hésité : $1 \%$ (103) alors qu'il y a $171 \%$ d'hésitants parmi les socialistes et $23: \%$ (22) parmi les électeurs «centristes 》 et «gaullistes》. Enfin, dans l'électorat de Jean Lecanuet, le plus fort pourcentage d'hésitants provient des électeurs « gaullistes »: $451 \%$ (28), alors qu'il n'est que de $27 \%$ (77) pour les électeurs «centristes» et de $28 \%$ (32) pour les électeurs «de gauche». 
Notes de Recherche

\section{Hesitations avant le scrutin et variables sociologiques}

$S E X E$

Pourcentage d'hésitants.

\begin{tabular}{|c|c|c|c|c|c|}
\hline & & & \multirow{2}{*}{$\begin{array}{c}\text { Vote premier tour } \\
\text { Mitterrand }\end{array}$} & \multirow{2}{*}{ Lecanuet } \\
\hline & & $\mathrm{De}$ & Gaulle & & \\
\hline Hommes & $\ldots \ldots$ & 10 & (238) & $11(166)$ & $33 \quad(85)$ \\
\hline Femmes & $\ldots \ldots$ & 16 & $(278)$ & $13(134)$ & $34 \quad(95)$ \\
\hline
\end{tabular}

Les femmes sont proportionnellement plus nombreuses que les hommes à avoir hésité avant de voter, quel que soit le candidat pour lequel elles ont effectivement voté. Cela se manifeste plus particulièrement dans le cas du vote pour le général de Gaulle : parmi les électrices du général de Gaulle qui ont hésité avant de voter, 39\% (44) ont hésité entre le général de Gaulle et Jean Lecanuet contre $70 \%$ (23) des hommes et $47 \%$ entre le général de Gaulle et François Mitterrand contre 9:\% des hommes. On a vu que les femmes ont moins fréquemment voté que les hommes pour François Mitterrand; mais pour les électrices qui ont hésité, François Mitterrand a été plus souvent à l'origine de l'hésitation que pour les hommes. Cet attrait plus grand de François Mitterrand sur les femmes est-il dû à sa personne ou à sa campagne électorale au cours de laquelle il s'est adressé à plusieurs reprises aux femmes (il a été le premier à soutenir le contrôle des naissances) ? Les éléments dont nous disposons ne permettent pas d'en décider. Les électrices de François Mitterrand qui ont hésité l'ont plus fait entre Mitterrand et Lecanuet $[41: \%$ (17)] que les hommes [5\% (19)]; les hommes ont plus hésité entre le général de Gaulle et François Mitterrand [42\% (19)] que les femmes [29:\% (17)].

$A G E$

Les électeurs qui ont moins de 43 ans sont proportionnellement plus nombreux à avoir hésité que ceux qui sont plus âgés, et cela quel que soit le candidat pour lequel ils ont voté.

Pourcentage d'hésitants

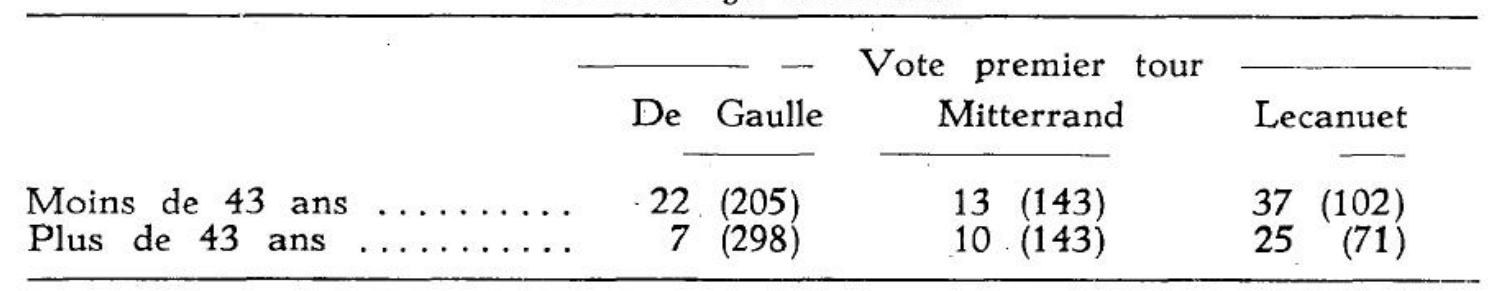




\section{Les Elections Présidenticlles}

Dans l'électorat du général de Gaulle, les plus jeunes hésitent plus entre le général de Gaulle et Jean Lecanuet $[51 \%$ (45) contre $45 \%$ (22) pour les plus âgés] qu'entre de Gaulle et Mitterrand [31\% (45) contrc $41 \%$ (22) four les plus de -13 ans].

Parmi les électeurs de Fransois Mitterrand, on rematque quiagmente avec l'âge l'hésitation entre de Giulle et Lecanuct [13\% (15) contre 0] et entre de Gaulle et Mitterrand (53\% contre 21\%), mais en revanche le nombre d'hésitants entre Mitterrand et Lecanuet diminue $[7 \%$ (15) contre $32 \%$ (19)].

Dans l'électorat de Jean Lecanuet, il y a peu de différences dues à l'âge, on peut noter toutefois une plus grande proportion d'hésit.tion entre Mitterrand et Lecanuet chez les plus jeunes [29:\% (38) contre 17:\% (18)].

\section{NIVEAII D'ETUDES}

L'hésitation avant le vote semble aller de pair avec l'intérêt politique; nous savons d'autre part que l'intérêt politique augmente arec le niveau d'études 11. Le t.ibleau suivant scmble confirmer ces hypothèses:

Pourcentage d'hésitants

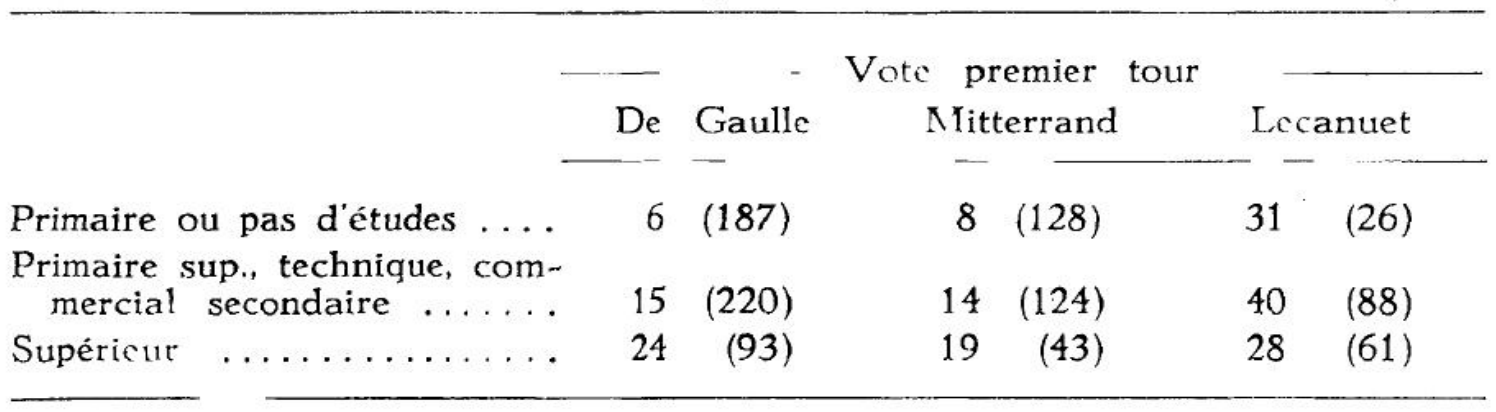

Le pourcentage d'hésitants croit avec le niveau d'études dans le groupe de ceux qui ont voté pour le général de Gaulle et pour François Mitterrand, mais il n'en est pas de même pour l'électorat de Jean Lecanuet: ses électeurs de niveau supérieur ont moins hésité que ceux de niveaux d'étude inférieurs. On pourrait expliquer ce phénomène par le fait que $251 \%$ de cette catégorie est constitué de femmes ayant fait des études supérieures mais n'exerçant aucune profession. La faiblesse des effectifs sur lesquels portent les calculs rendent difficile une analyse plus fine sur les candidats entre lesquels il y a eu hésitation.

11. Michelat (Guy), op. cit., p. 275. 


\section{Notes de Recherche}

\section{NIVEAU DE REVENUS}

L'hésitation croît avec le niveau de revenus passant de $8 \% \%$ (203) pour les électeurs de revenu mensuel inférieur à $1200 \mathrm{~F}$, à $18 \%$ (270) pour les électeurs de revenu supérieur. Ce résultat semblerait confirmer, comme celui constaté pour le niveau d'études, que l'hésitation est en relation avec l'intérêt politique, celui-ci augmente en effet avec le niveau de revenus 12.

\section{Pourcentage d'hésitants}

\begin{tabular}{|c|c|c|c|c|}
\hline \multirow{4}{*}{$\begin{array}{l}\text { Moins de } 1200 \mathrm{~F} \ldots \ldots \ldots \\
\text { Plus de } 1200 \mathrm{~F} \ldots \ldots \ldots\end{array}$} & & & \multirow{2}{*}{$\begin{array}{l}\text { Vote premier tour } \\
\text { Mitterrand }\end{array}$} & \\
\hline & $\mathrm{De}$ & Gaulle & & Lecanuet \\
\hline & 8 & (203) & (135) & (34) \\
\hline & 18 & $(270)$ & $(148)$ & $37 \quad(127)$ \\
\hline
\end{tabular}

En raison des faibles effectifs utilisés, aucune différence n'apparaît de façon claire en ce qui concerne les candidats entre lesquels il y a eu hésitation.

\section{PROFESSION}

C'est parmi les cadres moyens électeurs du général de Gaulle, les patrons de l'industrie et du commerce électeurs de François Mitterrand et parmi les professions libérales et cadres supérieurs électeurs de Jean Lecanuet qu'on rencontre la plus grande proportion d'hésitants.

\section{Pourcentage d'hésitants}

\begin{tabular}{|c|c|c|c|c|c|c|}
\hline \multirow{3}{*}{$\begin{array}{c}\text { Patrons de l'industrie et com- } \\
\text { merce } \quad . \ldots \ldots \ldots \ldots \ldots \ldots\end{array}$} & \multirow{3}{*}{$\begin{array}{r}\text { De } \\
8\end{array}$} & \multirow{3}{*}{$\begin{array}{c}\text { Gaulle } \\
(48)\end{array}$} & \multirow{2}{*}{\multicolumn{2}{|c|}{$\begin{array}{l}\text { Vote premier tour } \\
\text { Mitterrand }\end{array}$}} & \multirow{2}{*}{\multicolumn{2}{|c|}{$\overline{\text { Lecanuet }}$}} \\
\hline & & & & & & \\
\hline & & & 25 . & (20) & 25 & (12) \\
\hline Prof. lib., cadres sup. . . . . & 13 & (69) & 12 & (25) & 41 & (34) \\
\hline Cadres moyens $\ldots \ldots \ldots \ldots$ & 23 & (78) & 17 & (47) & 32 & (25) \\
\hline Employés $\ldots \ldots \ldots \ldots \ldots$ & 7 & (58) & 18 & (38) & 33 & (6) \\
\hline Ouvriers $\ldots \ldots \ldots \ldots \ldots \ldots$ & 10 & (77) & 5 & (86) & 21 & (19) \\
\hline Personnel de service $\ldots \ldots$ & 6 & (34). & 0 & (13) & 14 & $(7)$ \\
\hline Autres catégories $\ldots \ldots \ldots \ldots$ & 25 & (8) & 30 & $(7)$ & 75 & (4) \\
\hline Personnes non actives $\ldots \ldots$ & 13 & $(144)$ & 11 & (64) & 35 & (72) \\
\hline
\end{tabular}

12. Ibid. 


\section{LES MOTIVATIONS}

\section{Analyse des catégories}

L'analyse des motivations des votes aux deux tours de l'élection présidentielle porte sur l'exploitation de la question ouverte : "Quelles sont les prizipales raisons pour lesquelles rous avez voté pour ce iundidat?") qui suivait immédiatement la question: "Pouvez-tcus me dire pour quel candiclat vori: avez roté ?"Seuls ont été envisagés les quatre candidats arrivés en tête le 5 décembre.

Les classifications quelque peu schématiques qui ont été utilisées et qui sembleront parfois arbitraires ou artificielles étaient rendues néccssaires par l'exploitation mécinographique. C'est pourquoi il a semblé utile de préciser quel contenu elles recouvrent.

Il est évident que les «motivations 》 ainsi recueillies sont des motivations déclarées et qu'il peut exister un certain écart entre elles et les déterminants profonds du vote.

- Les motivations du vote pour le général de Gaulle 13

- Stabilité. sécurité.

I.e thème concerne les notions de stabilité, de sécurité, d'habitude, de peur de l'inconnu. Le mot «stabilité 》 revient plusieurs fois, soit seul, soit dans des expressions du type : «il a maintenu la stabilité 》, «sa politique nous a ramené de l'ordre, de la stabilité ». Les électeurs évoquent également le fait qu' "avec le général l'on sait où l'on va » ou que «c'est lui qui nous donnait le plus de sécurité ». La satisfaction peut ètre plus générale : «je me trouve satisfaite de son régime», «on a été content de le trouver, il a fait de bonnes choses", «les choses ront mienx ». A défaut de satisfaction. l'habitude motive le vote pour le génér.ul de Gaulle «parce qu'on est habitué comme ça » et «parce que I'on a peur de l'aventure représentéc par «les autres» dont on reut éviter la venue ». On sait ce qu'on a, on ne sait pas ce qu'on perd»; «on sait ce qu'il a fait, on ignore ce que feront les autres»; «on a encore besoin de lui, il faut qu'il puisse continuer ».

13. On peut se reporter à plusieurs études antérieures concernant l'attitude à l'égard du général de Gaulle : cf. en particulier Converse (Philip), Dupeux (Georges), "Eisenhower et de Gaulle. Les généraux devant l'opinion 》, Revue française de science politique 12 (1), 1962, pp. 54-92; Dogan (Mattei), «Le personnel politique et la personnalité charismatique», Rer'ue francaise de sociologie 6 (3), 1965, pp. 305-324; Mickielat (Guy). op. cit., pp. 244 sqq. 


\section{Notes de Recherche}

Le seul «valable 》.

Cette motivation exprime la croyance en la supériorité du général de Gaulle : d'une part, il est «le seul valable», «le mieux», 《le seul susceptible de faire quelque chose de réel »; d'autre part, les autres candidats «étaient vraiment trop politicards», «n'étaient absolument pas valables» et «la coalition contre lui était trop malpropre».

\section{De Gaulle est un grand homme.}

Cette catégorie concerne les qualités personnelles du général de Gaulle sur le plan de l'honnêteté, de la «classe», et de l'autorité : c'est «le plus propre», et c'est 《un homme droit, sincère», «il tient ses promesses », «c'est l'intérêt général qui le guide », 《on a confiance en lui »; c'est 《un homme de poigne et de tête», «un grand homme», «un grand soldat ».

\section{- Sa politique extérieure: le prestige de la France.}

Cette motivation traduit une forte sensibilisation au prestige actuel de la France dû à la politique extérieure du général de Gaulle. «On était vraiment tombé bien bas», mais «depuis que le général de Gaulle est au pouvoir, la France a remonté énormément son prestige à l'étranger ». Avant de Gaulle, la France était humiliée, déconsidérée et sans poids, elle joue maintenant un rôle très important sur le plan international : « elle est indépendante ». Certains éléments particuliers de la politique extérieure - Vietnam, relations avec U.R.S.S., aide aux pays sous-développés - sont mentionnés comme «une bonne chose».

\section{Reconnaissance.}

On doit de la reconnaissance au chef de l'Etat soit d'une manière générale (《 on lui doit tout 》), soit en fonction de périodes particulières : « il nous a sortis de l'occupation 》, «l'homme du 18 juin », « il nous a sauvé du marasme en $46.47 》$, «la fin de la guerre d'Algérie», «la remise en ordre du pays qu'il a ramené de la pagaille $\gg$.

\section{Nationalisme, patriotisme.}

Les réponses dessinent l'image d'un homme qui agit essentiellement en fonction de la France et qui est «un grand Français». D'une part, de Gaulle «a fait beaucoup de bien à la France», « a temonté la France »; d'autre part, c'est «un bon Français» à la différence des autres qui sont attirés par «le gain et l'apport qu'on leur donne ». Il est «français» au sens où l'est un bon patriote agissant en fonction des seuls intérêts et aspirations de son pays. 
- Contre la Quatrième République.

Cette motivation manifeste l'opposition à «l'ancien système» et aux «anciens partis». On a voté pour de Gaulle «parce qu'il n'est pas l'esclave des partis », parce que « depuis qu'il est là, finis les changements de gouvernement 》, «pour ne pas retourner aux anciens systèmes 》, «contre ces gouvernements qui nous ont menés là où on était ». Il semble que ces motivations soient formulées de façon assez précise.

- Sa politique économique.

$\mathrm{Ce}$ thème concerne la politique économique, soit dans son ensemble : « de Gaulle arrange la vie mieux 》, soit dans des domaines particuliers : " pour les vieux», «moins de grèves», «pour la stabilité financière de la France ».

\section{- Divers.}

Cette catégorie regroupe les réponses inclassables ailleurs, «avec l'espoir qu'il nous débarrassera des gaullistes 》, "pour éviter une réaction à droite », «bien qu'il ne fasse rien pour la classe ouvrière ».

Motivations du vote an second tour.

On a retenu pour le second tour les catégories du premier tour en substituant ̀̀ la seconde ( $\ll$ de Gaulle est le seul valable »), l'opposition à Mitterrand : «Mitterrand ne me plaît pas »; « je me moque des partis et de M. Mitterrand»; «je ne voulais absolument pas voter pour M. Mitterrand».

\section{- Les motivations du vote pour François Mitterrand.}

- Il est plus proche des ouvriers.

Mitterrand est «pour les ouvriers», c'est lui qui « aurait défendu le plus les ouvriers», qui «semblait être le plus capable de soutenir un peu l'ouvrier »; « je crois qu'il se tapproche des désirs de la vie ouvrière ». Ces réponses traduisent souvent de surcroît une opposition au général de Gaulle qui «a promis, n'a rien fait pour la classe ouvrière »; Mitterrand semble être "plus pour la classe ouvrière que de Gaulle ».

\section{- Contre de Gaulle.}

Cette opposition se situe à trois niveaux et tout d'abord celui d'un mécontentement provoqué par la personnalité du général de Gaulle (on veut «ramener de Gaulle à plus de modestie», on a une «rancune personnelle contre de Gaulle»). Une déception plus accusée provoque un désir 


\section{Notes de Recherche}

de changement; les électeurs votent alors pour François Mittetrand «pour que ça change », en pensant que «ça irait mieux». Enfin, le mécontentement s'accompagne parfois d'un vote tactique: il s'agit alors de voter pour le candidat le plus favorisé, parce que c'est celui «qui a le plus de chances de mettre de Gaulle en ballottage ", "c'est le seul capable de rassembler une majorité ».

Contre la force de frappe.

Cette motivation traduit l'opposition de certains électeurs à la force de frappe avec laquelle ils ne sont «pas d'accord». Ils refusent que «l'on dépense des milliards pour la bombe atomique».

C'est le condiditt die la gauche.

Lcs électeurs expliquent leur vote par leurs idées personnelles, leurs attitudes propres : « parce que j'ai des idées socialistes», « $j$ 'ai le cœur assez iz gauche », «je suis plutôt de gauche», « $j$ 'ai roté pour le candidat qui représentait la gauche ». Enfin apparaît la discipline et l'unité de la gauche : «notre parti nous le disait; nous, nous sommes disciplinés 》; «union de la gauche»; «rassemblement de la gauche ».

\section{C'est. un démorrate.}

François Mitterrand «est un grand démocrate», il offre une «véritable définition de la démocratie» et il est «pour le droit de grève» et «pour la liberté d'expression».

Parce qu'il est jenne.

«Il faut voter pour un jeune" par opposition au général de Gaulle et "parce que de Gaulle a énormément vieilli et qu'en fin de septennat il aurait $\$ 2$ ans $\gg$.

- Parce que de Gaulle c'est le pouvoir personnel.

Ces réponses expriment une opposition à la conception gaulliste du pouvoir sur deux plans, celui de l'opposition au général de Gaulle Jui-même parce qu' «il se prend un peu pour le bon Dieu», «Mitterrand est moins autoritaire» et celui de l'opposition à la notion même de pouroir personnel : on est «résolument contre le pouvoir personnel 》, «opposé -au pouvoir personnel, question de principe».

Sa politique ćconomique et sociale.

On constate d'une part un accord avec la «politique sociale 》 du candidat, d'autre part unc opposition dans ce domaine au président de la 


\section{Les Elections Présidentielles}

République en exercice: Mitterrand «se pencherait sur le social plus que le général de Gaulle qui plane un peu »; il serait "plus pour les œuvres sociales que le gouvernement actuel». On attend de Mitterrand des réalisations dans des domaines particuliers : «les vieilles personnes pour lesquelles on ne fait pas assez d'effort », les jeunes, le logement... On espère «un changement social 》: «c'est seulement avec ces hommes que nous pouvons faire une France nouvelle, faire une politique sociale».

\section{Il est le plus proche de mes idées.}

Ce thème concerne la concordance entre les idées de Mitterrand et celles de ses électeurs : le candidat convient à l'électeur, correspond à « ses idées》, 《il représente mes opinions politiques》, c'est lui qui «se rapproche le plus de mes idées ». L'électeur est attiré par son programme, ses projets; sa conception de la vie politique: «j'estime qu'il a exposé un programme très précis, comportant assez de nouveauté pour engager une action supplémentaire nous faisant avancer ».

\section{Divers :}

Cette catégorie regroupe les réponses difficilement classables ailleurs et qui expriment un désir de changement non précisé, un attrait pour la personnalité de Mitterrand ( il me plaît»), des réserves sur le candidat (《il a derrière lui plusieurs groupements qui peuvent le surveiller »), ou l'influence de tiers (« $j$ 'ai suivi les opinions de mon mari»).

Motivations du vote an second tour.

Aux catégories utilisées pour l'analyse du premier tour, on a ajouté le thème : "pour donner une leçon au général de Gaulle ». "On savait bien qu'il passerait, mais il fallait qu'il comprenne. 》 On a voté pour Mitterrand «parce que ça lui faisait pas de mal à de Gaulle de voir qu'on lui résistait ».

\section{LES MOtIVATIONS DU VOTE pour JEAN Lecanuet}

Son programme.

Les réponses regroupées dans cette catégorie concernent le programme de Jean Lecanuet, ses idées «centristes», ou perçues comme centristes: Le programme apparaît dans les réponses du type : «il se rapprochait le plus de mes idées 》, "parce qu'il représentait mes opinions 》. Les termes employés restent imprécis et semblent traduire une adhésion assez. forte, libre de problèmes et de questions. L'élément qui a donné lieu aux plus longues réponses montre un attachement profond, par-delà les 


\section{Notes de Recherche}

«opinions» et les «programmes》, à une idéologie traditionnelle du centre : «j'avais l'impression qu'il était moyen, que ce n'était pas un extrémiste, ni d'un côté, ni de l'autre»; 《il ressemblait à la démocratie américaine »; " parce que j'aime bien un bon milieu, ni d'un côté, ni de l'autre ". On retrouve ici la conception d'une vie politique pondérée, à mi-chemin entre la gauche et la droite, et exempte de tout excès.

\section{- L'Europe.}

Les réponses regroupées sous ce thème traduisent un attachement (souvent vague) à l'idée d'Europe. Les expressions qui reviennent le plus souvent sont brèves : "pour l'Europe »: "son idée euroféenne ». Quelques-unes considèrent le problème européen en fonction de la politique gaulliste et sont par là même plus précises: "le général de Gaulle au point de vue de l'Europe ne répond plus à ce qu'on attend d'un président »; «incertitude laissée sur le plan européen, le manque de définition du programme gaulliste».

$$
\text { Il. est jezne. }
$$

L'adjectif «jeune » est mentionné seul ou dans des expressions du type : «j'aurais aimé que la France ait un président jeune». La jeunesse de Lecanuet a également été opposée à l'âge avancé du président en fonction: «je pense que de Gaulle devra laisser la place aux jeunes», « l'ouvrier doit bien prendre s.l tetraite à 60 ans, de Gaulle aussi». Il esit possible que l'attrait exercé par l'âge de Jean Lecanuet ne soit qu'une conséquence d'une hostilité générale à toute gérontocratic. même si le général de Gaulle n'est pas explicitement mentionné.

\section{Contre de Gaulle.}

Cettc catégorie concerne thostilité au gaullisme accompagné parfois d'unc crainte de l'après-gaullisme. Au premier plan, on trouve un refus global du général de Gaulle, soit exprimé de façon très simple : " pour ne pas voter pour de Gaulle»; soit motivé par un mécontentement dans un domaine particulier. On veut au moins «mettre de Gaulle en ballottage ». Enfin le vote pour Jean Lecanuet apparaît la seule alternative possible à un vote pour le général de Gaulle : "je voulais remplacer de Gaulle et les autres ne correspondaient pas à mes opinions ». A la limite, si l'on accepte le général de Gaulle, on craint qu'il n'achèrc pas son septennat : « de Gaulle ne va pas continuer, et il va nous en coller un autre».

\section{Ses qualités personnelles.}

«Son dynamisme »; «sa franchise»; « son honnêteté »; «son intelli. gence »; 《il a du cran». 
- Le mieux pour la France.

- Il faut «rajeunir la politique».

- Politique intérieure.

«Politique économique et sociale»; «pour les jeunes»; «pour l'éducation nationale ».

- Divers.

«Contre Mitterrand»; «contre les communistes»; « il veut faire la politique de de Gaulle sans de Gaulle »; «Mitterrand est franc-maçon ».

Motivations du vote pour Jean-Louis Tixier-Vignancour

- Pour la France.

«Le prestige de la France.»

- A cause de l'Algérie.

«J'espérais que les portes des prisons allaient s'ouvrir»; «il était franchement pour l'amnistie »; «c'est lui qui a le plus défendu le problème des rapatriés ».

- Son programme.

Cette catégorie regroupe les réponses concernant son programme, «qui était raisonnable au point de vue financier»; 《ses idées conformes à ce qu'il a toujours fait »; "celui qui se rapproche le plus de mes idées 》; «j'étais de l'Action française, Ini aussi ». C'est aussi dans cette catégorie que sont rangées les réponses du type : «il défendait le petit commerce ».

- C'était le plus antigaulliste.

«Depuis quatre ans, on était d'accord sur ce qu'il disait au sujet du général de Gaulle. » On a voté pour lui «parce que c'était le fer de lance de l'opposition» et «pour faire front à de Gaulle».

- Ses qualités personnelles.

«Il avait l'air propre»; il était «le plus juste et le seul valable». 
Notes de Recherche

Contre liside auxi pays sous-dételoppés.

Divers.

"Origines béarnaises, pas pour ses idées mais parce que je suis de son pays»; "pour que ça change»: "parce qu'il y a un rapport avec Kennedy 》.

\section{Importance des diverses motivations}

Les électeurs ont donné des raisons de vote plus nombreuses au premier tour qu'au second. Il semble qu'au premier tour on ait exprimé tout d'abord ses préférences personnelles et ses appréciations sur la personnalité des candidats. Au second tour, il s'est agi de choisir un président, de faire un acte plus politique et le vote a été expliqué de manière plus catégorique et moins détaillée.

\section{Motriations de vote pour le général de Gaulle.}

$34 \%$ des électeurs ont voté au premier tour motivés par le désir de stabilité, $27 \%$ ont voté parce que le général de Gaulle était « un grand homme», $21 \%$ parce qu'il était «le plus valable»; $17 \%$ ont été motivés par la reconnaissance.

$\mathrm{Au}$ second tour, la stabilité reste déterminante, mais dans une moindre proportion: 25 'són des électeurs ont voté pour cette raison. L'un des éléments importants du vote a été l'opposition à François Mitterrand: 19:\% des électeurs expliquent ainsi leur vote. La décision semble donc avoir été plus politique qu'au premier tour. Les autres motivations importantes au second tour sont les mêmes qu'au premier mais chaque fois dans une moindre proportion.

\section{Motivitions du vote pour Françols Mitterrand}

$\mathrm{Au}$ premier tour, cinq raisons ont plus particulièrement motivé les électeurs. Les quatre premières concernaient une idéologie et un programme de gauche, la cinquième l'opposition au général de Gaulle $18 \%$ des électeurs). Au second tour, cette opposition concerne $29 \%$ des électeurs. En effet, les opposants à de Gaulle ayant voté au premier tour pour un autre candidat se sont talliés à Mitterrand. Il n'y a plus que 12\% des électeurs qui ont voté pour lui «parce qu'il était plus proche des ouvriers (21 $\sigma_{\%}^{\prime}$ au premier tour); et $13 \%$ ont voté parce que c'était «le candidat de la gauche» (contre 19\% au premier tour). 
Motivations du vote pour le général de Gaulle

\begin{tabular}{|c|c|c|}
\hline & 1er tour & $2^{e}$ tou \\
\hline 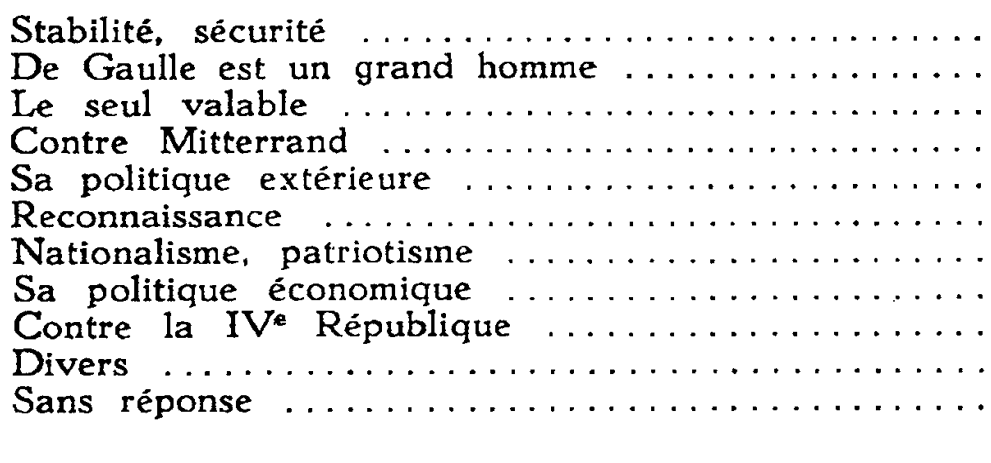 & $\begin{array}{r}14 \\
17 \\
12 \\
5 \\
3 \\
9 \\
3 \\
(516)\end{array}$ & $\begin{array}{r}19 \\
9 \\
10 \\
9 \\
4 \\
4 \\
9 \\
10 \\
(627)\end{array}$ \\
\hline
\end{tabular}

Motivations du vote pour François Mitterrand

Il est plus proche des ouvriers $\ldots \ldots \ldots \ldots \ldots \ldots$

Sa politique économique et sociale $\ldots \ldots \ldots \ldots \ldots$.

Il est le plus proche de mes idées $\ldots \ldots \ldots \ldots \ldots \ldots$

C'est le candidat de la gauche $\ldots \ldots \ldots \ldots \ldots \ldots$

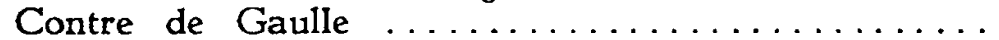

Pour donner une leçon à de Gaulle ............

Contre la force de frappe ..................

Parce qu'il est jeune $\ldots \ldots \ldots \ldots \ldots \ldots \ldots \ldots \ldots$

C'est un démocrate $\ldots \ldots \ldots \ldots \ldots \ldots \ldots \ldots \ldots$

Parce que de Gaulle c'est le pouvoir personnel ....

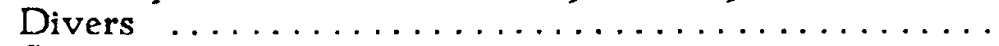

Sans rêponse

$\begin{array}{rr}21 & 12 \\ 21 & 20 \\ 20 & 18 \\ 19 & 13 \\ 18 & 29 \\ & 2 \\ 9 & 5 \\ 7 & 4 \\ 4 & 5 \\ 4 & 4 \\ 17 & 14 \\ 2 & 8\end{array}$

(300) (376)

Motivations du vote pour Jean Lecanuet

Son programme $\ldots \ldots \ldots \ldots \ldots \ldots \ldots \ldots \ldots$

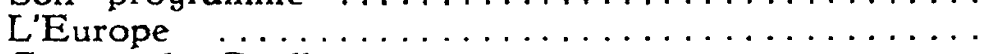

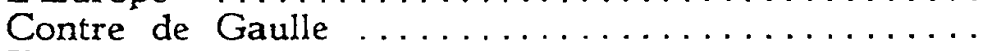

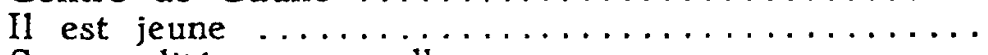

Ses qualités personnelles $\ldots \ldots \ldots \ldots \ldots \ldots \ldots \ldots$

Sa politique intérieure $\ldots \ldots \ldots \ldots \ldots \ldots \ldots \ldots$.

Il faut rajeunir la politique $\ldots \ldots \ldots \ldots \ldots \ldots \ldots$

Divers $\ldots \ldots \ldots \ldots \ldots$

Motivations du vote pour Jean-Louis Tixier-Vignancour

A cause de l'Algérie $\ldots \ldots \ldots \ldots \ldots \ldots \ldots \ldots$

C'est le plus antigaulliste $\ldots \ldots \ldots \ldots \ldots \ldots \ldots \ldots$

Son programme $\ldots \ldots \ldots \ldots \ldots \ldots \ldots \ldots \ldots \ldots \ldots$

Ses qualités personnelles $\ldots \ldots \ldots \ldots \ldots \ldots \ldots \ldots$

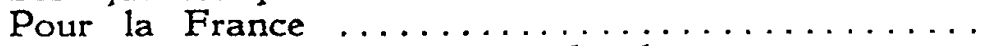

Contre l'aide aux pays sous-développés $\ldots \ldots \ldots$.

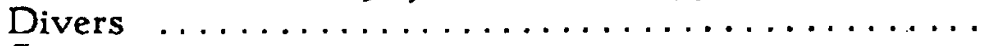

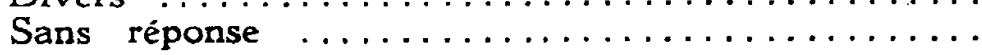




\section{Notes de Recherche}

\section{- Motivations du vote porrr Jean Lecanuft}

Les éléments les plus importants pour le vote ont été le programme centriste développé par le candidat $(29 \%)$, sa politique européenne (29\%) et l'opposition au général de Gaulle (28, (c) $)$. Ia jeunesse du candidat a été inentionnée par $24 \%$ de ses électeurs.

Motivations du vote pour Jean-Louis Tixier-Vignancour

$40 \%$ des électeurs de Jean-Louis Tixier-Vignancour ont voté pour lui à cause de l'Algérie, $28 \%$,o parce qu'il était le candidat le plus antigaulliste et $28 \%$ i cause de son programme.

\section{Motivations du vote et hesitations avant le scrutin}

On peut considérer que les motivations données au vote sont en relation avec les hésitations que l'on a eues avant de roter. Or on constate que certaines des motivations analysées ne sont pas représentées d'égale façon dans le groupe des hésitants et dans le groupe des non-hésitants. Dins l'électorat du général de Gaulle. les motivitions «stabilité 》, «c'est le seul valable ». "la politique extérieure du général de Gaulle assure le prestige de la France» sont plus fréquentes chez les hésitants, et les motivations «c'est un grand homme», « reconnaissance » et 《nationalisme » plus fréquentes chez les non-hésitants.

\begin{tabular}{|c|c|c|}
\hline $\begin{array}{c}\text { Motivations } d u \text { vote 1er tour } \\
\mathrm{G}^{u 1} \mathrm{DE} \text { GAULLE }\end{array}$ & Hésitants & Non-hésitants \\
\hline 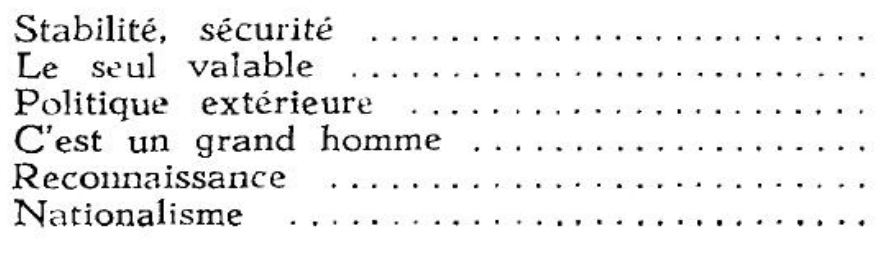 & $\begin{array}{r}48 \\
33 \\
18 \\
13 \\
9 \\
4 \\
(67)\end{array}$ & $\begin{array}{c}32 \\
19 \\
13 \\
29 \\
18 \\
13 \\
(447)\end{array}$ \\
\hline
\end{tabular}

C'est-à-dire qu'il semble que les non-hésitints soient plus sensibles à des thèmes affectifs liés à l'attachement personnel au général de Gaulle : «je vote pour le général de Gaulle parce que c'est lui, parce que c'est un grand homme, ça ne se discute pas », alors que les hésitants qui ont en définitive voté pour le général de Gaulle l'ont fait pour des raisons plus politiques, «qui se discutent»: «stabilité politique», «comparé aux 


\section{Les Elections Présidentielles}

autres, c'est le seul homme politique valable.» et : sa politique extérieure assure le prestige et l'indépendance de la France ».

Nous retrouvons des phénomènes semblables en ce qui concerne les motivations de vote pour François Mitterrand : les non-hésitants invoquent le fait que le candidat pour lequel ils ont.voté est «le plus proche des ouvriers », « le plus .proche de leurs idées »; : « on est de gauche, il est le candidat de la gauche, . donc on vote pour lui ». Les hésitants optent pour lui. parce qu'il est «contre la force de frappe».

\begin{tabular}{|c|c|c|}
\hline $\begin{array}{c}\text { Motivations du vote } 1 \text { er tour } \\
\text { F. MITTERRAND }\end{array}$ & Hésitănts & $\therefore$ Non-hésitants \\
\hline $\begin{array}{l}\text { Il est plus proche des ouvriers } \ldots \ldots \ldots \ldots \ldots \\
\text { Il est plus proche de mes idées } \ldots \ldots \ldots \ldots \ldots \\
\text { Contre la force de frappe } \ldots \ldots \ldots \ldots \ldots\end{array}$ & $\begin{array}{c}6 \\
3 \\
14 \\
(36)\end{array}$ & $\begin{array}{r}21 \\
14 \\
5 \\
(264)\end{array}$ \\
\hline
\end{tabular}

En ce qui concerne les raisons du vote pour Jean Lecanuet, la distinction entre thèmes politiques et thèmes affectifs correspondant à l'hésitation et à la non-hésitation semble moins évidente puisque les hésitants ont voté pour Jean Lecanuet parce qu'il est jeune et « pour ses qualités personnelles », alors que les non-hésitants sont plus sensibles au thème «son programme». Ils marqueraient ainsi leur adhésion à une idéologie centriste traditionnelle.

\begin{tabular}{|c|c|c|}
\hline $\begin{array}{c}\text { Motivations du vote } 1^{\text {er }} \text { tour } \\
\text { J. LECANUET }\end{array}$ & Hésitants & Non-hésitants \\
\hline $\begin{array}{l}\text { Son programme } \ldots \ldots \ldots \ldots \ldots \ldots \\
\text { Il est jeune } \ldots \ldots \ldots \\
\text { Ses qualités personnelles } \ldots \ldots \ldots \ldots\end{array}$ & $\begin{array}{c}15 \\
30 \\
13 \\
(60)\end{array}$ & $\begin{array}{r}36 \\
21 \\
8 \\
(118)\end{array}$ \\
\hline
\end{tabular}

En ce quii concerne les électorats du général de Gaulle et de François Mitterrand du moins, il semblerait que la non-hésitation exprime une adhésion globale soit à un homme, soit à une idéologie et que l'hésitation corresponde à un point de vue plus politique.

Existe-t-il des différences de motivation de vote au premier tour suivant les candidats entre lesquels on a hésité ?

En ce qui concerne les électeurs qui ont en définitive choisi de Gaulle au premier tour, on constate que ceux qui ont hésité entre de Gaulle et Lecanuet ont été plus sensibles au thème «sécurité, stabilité 》 et moins 


\section{Notes de Recherche}

aux thèmes «c'est le seul valable » et «c'est un grand homme » que ceux qui ont hésité entre de Gaulle et Mitterrand.

On pourrait dire, si on suppose qu'il existe un rapport entre motivation du vote réel et hésitation avant le rote, qu'on a hésité entre de Gaulle et Lecanuet parce que l'un incarnait plus la stabilité et que l'autre avait un programme plus satisfaisant (sur I'Europe en particulier) et qu'on a hésité entre de Gaulle et Mitterrand parce que le second était le candidat de la gauche mais que le premier était «un grand homme» et «le seul valable».

Hésitants au premier tour et votants pout de Gaulle

\begin{tabular}{|c|c|c|}
\hline Motivations du tote $1^{\text {er }}$ tour & $\begin{array}{l}\text { Entre de Gaulle } \\
\text { et Mitterrand }\end{array}$ & $\begin{array}{l}\text { Entre de Gaulle } \\
\text { et Lecanuet }\end{array}$ \\
\hline $\begin{array}{l}\text { Stabilité, sécurité } \ldots \ldots \ldots \ldots \\
\text { Le seul valable } \ldots \ldots \ldots \ldots \ldots \\
\text { C'est un grand homme } \ldots \ldots \ldots\end{array}$ & $\begin{array}{c}39 \\
35 \\
22 \\
(33)\end{array}$ & $\begin{array}{c}61 \\
24 \\
12 \\
(23)\end{array}$ \\
\hline
\end{tabular}

La faiblesse des effectifs ne permet guère d'analyser les motivations des hésitants qui ont voté pour François Mitterrand et Jean Lecanuet. On peut cependant faire l'hypothèse que parmi les électeurs de Jean Lecanuet, ceux qui ont hésité entre Mitterrand et Lecanuet ont été plus sensibles au programme de ce dernier $[25 \%$ (16) $]$ et moins sensibles à ses «qualités personnelles 》 $[6 \%$ (16) $]$ que ceux qui ont hésité entre de Gaulle et Lecanuet (respectivement $6 \%$ et 16:\%). C'est-à-dire que ceux qui ont hésité entre Lecanuet et de Gaulle ont été plus sensibles aux qualités personnelles et ceux qui ont hésité entre Lecanuet et Mitterrand l'ont été plus aux problèmes de programme (en particulier l'Europe).

\section{Motivations du vote et preférences politiques}

\begin{tabular}{|c|c|c|c|}
\hline $\begin{array}{c}\text { Motivations du vote ler tout } \\
\mathrm{G}^{\mathrm{a}} \mathrm{DE}_{\mathrm{DA}} \mathrm{DAULL}\end{array}$ & $\begin{array}{l}\text { «Gaullistes } \\
\text { (U.N.R.-U.D.T., } \\
\text { rép. indép.) }\end{array}$ & $\begin{array}{l}\text { "Centristes» } \\
\text { (M.R.P., C.N.I.) }\end{array}$ & $\begin{array}{l}\text { "Gauche» } \\
\text { (P.C.., P.S.U., } \\
\text { S.F.I.O., rad.) }\end{array}$ \\
\hline 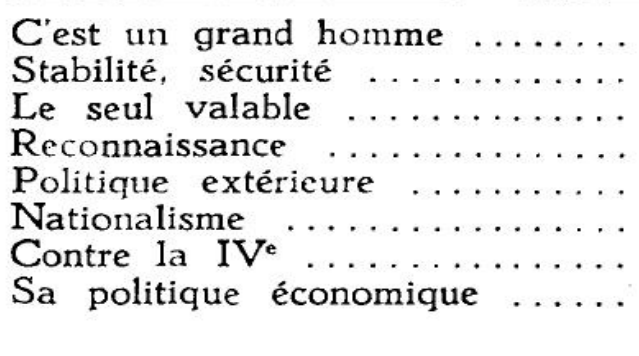 & $\begin{array}{r}32 \\
29 \\
23 \\
19 \\
15 \\
14 \\
5 \\
3 \\
(265)\end{array}$ & $\begin{array}{r}23 \\
44 \\
25 \\
8 \\
19 \\
10 \\
2 \\
2 \\
(52)\end{array}$ & $\begin{array}{r}21 \\
34 \\
14 \\
27 \\
14 \\
14 \\
4 \\
7 \\
(56)\end{array}$ \\
\hline
\end{tabular}




\section{Les Elections Présidentielles}

$\mathrm{Si}$ on examine quelles sont les motivations spécifiques des trois types d'électorat : "gaulliste 》, " centriste 》, " gauche », c'est-à-dire si on isole les motivations les plus représentées dans un électorat relativement aux autres, on voit que le gaullisme affectif, l'attachement personnel au général de Gaulle ("c'est un grand homme») est le thème essentiel pour les gaullistes alors que c'est «la stabilité » qui a le plus motivé «la gauche » et surtout les «centristes ». On pourrait donc dire que c'est surtout la peur de l'instabilité qui a poussé l'opposition à voter pour de Gaulle. Les électeurs «centristes 》 ont été les plus sensibles à la politique extérieure du général de Gaulle, c'est-à-dire que pour eux les problèmes européens ont dû paraître secondaires. Les électeurs «de gauche » qui ont voté pour le général de Gaulle l'ont fait, plus que les électeurs d'autres partis, par «reconnaissance 》 («après ce qu'a fait de Gaulle pendant la guerre $39-45$ et en finissant la guerre d'Algérie ») et autant que les gaullistes par «nationalisme »; c'est-à-dire en reprenant la distinction que nous avons déjà utilisée, pour des raisons plutôt affectives. Il est intéressant de noter que la politique extérieure ne semble pas avoir été un thème dominant, il est même moins important que pour les «centristes ».

Etant donné l'accord qui existe entre l'électorat théorique de Mitterrand et son électorat réel, les effectifs des électeurs «centristes 》 et " gaullistes 》 sont trop faibles pour qu'on puisse en comparer les motivations de vote pour François Mitterrand.

Mais pour Jean Lecanuet des différences apparaissent :

\begin{tabular}{|c|c|c|c|}
\hline $\begin{array}{c}\text { Motivations } \\
\text { du vote 1er tour } \\
\text { J. LECANUET }\end{array}$ & $\begin{array}{l}\text { «Gaullistes » } \\
\text { (U.N.R.-U.D.T., } \\
\text { Rép. indép.) }\end{array}$ & $\begin{array}{l}\text { «Centristes } \\
\text { (M.R.P., C.N.I.) }\end{array}$ & $\begin{array}{l}\text { «Gauche» } \\
\text { (P.C.,P.S.U.., } \\
\text { S.F.I.O., rad.) }\end{array}$ \\
\hline $\begin{array}{l}\text { A cause de l'Europe } \ldots \ldots \ldots \ldots \\
\text { Contre de Gaulle } \ldots \ldots \ldots \ldots \ldots \\
\text { Son programme } \ldots \ldots \ldots \ldots \\
\text { Parce qu'il est jeune } \ldots \ldots \ldots \\
\text { Ses qualités personnelles } \ldots \ldots\end{array}$ & $\begin{array}{c}4 \\
39 \\
18 \\
29 \\
18 \\
(40)\end{array}$ & $\begin{array}{c}34 \\
32 \\
27 \\
18 \\
6 \\
(77)\end{array}$ & $\begin{array}{l}25 \\
16 \\
34 \\
31 \\
16 \\
(32)\end{array}$ \\
\hline
\end{tabular}

La politique européenne est la motivation essentielle du vote des «centristes » pour Jean Lecanuet; elle joue aussi un rôle important pour les électeurs «de gauche»; par contre, elle joue un rôle minime pour les " gaullistes 》, contrairement à ce qu'on aurait pu penser; pour ces derniers, c'est l'opposition au général de Gaulle qui jouerait le rôle déterminant. $\mathrm{La}$ jeunesse du candidat et ses qualités personnelles jouent un rôle plus important pour les «gaullistes 》 et les électeurs «de gauche» que pour les «centristes», comme si ceux-ci votaient plus en fonction de l'attachement à l'idéologie centriste où les thèmes de «l'Europe » et de «l'op- 


\section{Notes de: Recherche}

position à de Gaulle» pouvaient avoir comme facteur commun l'anticommunisme; par contre, les électeurs gaullistes et « de gauche » auraient été plus sensibles à l'homme Lecanuet comparé à leurs candidats théoriques : de Gaulle et Mfitterrand, ce dernier ayant aussi l'inconvénient de s'être allié aux communistes. Pour les électeurs de gauche, l'importance du thème européen qui aurait alors une signification anticommuniste pourrait confirmer cette dernière hypothèse.

\section{Motivations du vote et variables sociologiques}

Les électrices de Jean Lecanuet ont été plus sensibles que ses électeurs à des motivations liées à la personne même du candidat : « jeunesse » et «qualités personnelles》: elles sont également plus nombreuses à avoir voté par antigaullisme ( $32 \%$ d'entre elles contre $2.4 \%$ des hommes). Les électeurs ont plutôt voté en fonction du programme européen et de la politique intérieure proposés par Jean Lecanuet:

\begin{tabular}{|c|c|c|}
\hline $\begin{array}{c}\text { Motivations diu vote ler tour } \\
\text { J. LECANUET }\end{array}$ & Hommes & Femmes \\
\hline 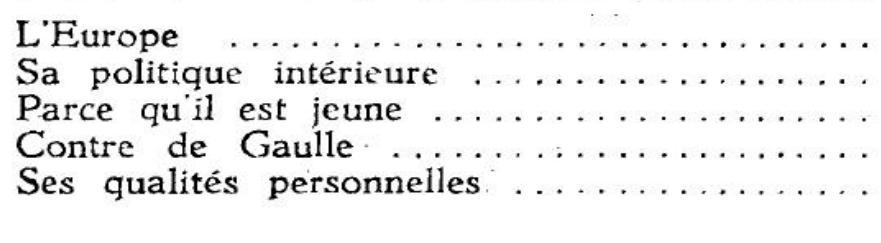 & $\begin{array}{c}35 \\
13 \\
25 \\
24 \\
7 \\
(85)\end{array}$ & $\begin{array}{c}22 \\
4 \\
34 \\
32 \\
13 \\
(94)\end{array}$ \\
\hline
\end{tabular}

On peut faire des remarques du même type à propos de l'électorat de François Mitterrand : $10 \%$ des électrices ont voté pour lui parce qu'il était jeune, contre $4 \% \%$ des électeurs. Elles ont moins tenu compte des éléments proprement politiques et idéologiques tels que : «c'est le candidat de gauche» et «l'opposition au pouroir personnel»:

\begin{tabular}{ccc}
\hline $\begin{array}{c}\text { Motivations du vote fer tour } \\
\text { F. MITTERRAND }\end{array}$ & Hommes & Femmes \\
\hline C'est le candidat de la gauche $\ldots \ldots \ldots \ldots \ldots \ldots$ & 22 & 16 \\
Il est jeune $\ldots \ldots \ldots \ldots \ldots \ldots \ldots \ldots \ldots \ldots$ & $\begin{array}{c}4 \\
7\end{array}$ & 10 \\
De Gaulle, cest le pouvoir personnel $\ldots \ldots \ldots$ & $(166)$ & $(134)$ \\
\hline
\end{tabular}




\section{Les Elections Présidentielles}

Ces résultats se retrouvent au second tour; s'y ajoute la différence concernant le thème «sa politique économique et sociale » citée par $24 \%$ (208) des hommes et par seulement $15 \%$ (168) des femmes.

$A G E$

\begin{tabular}{|c|c|c|}
\hline $\begin{array}{c}\text { Motivations du vote } 2^{e} \text { tour } \\
\text { J. LECANUET }\end{array}$ & $\begin{array}{l}\text { Moins } \\
\text { de } 43 \text { ans }\end{array}$ & $\begin{array}{l}\text { Plus } \\
\text { de } 43 \text { ans }\end{array}$ \\
\hline 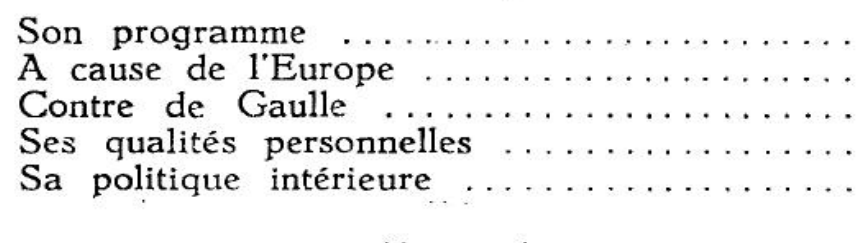 & $\begin{array}{c}26 \\
34 \\
31 \\
9 \\
11 \\
(102)\end{array}$ & $\begin{array}{c}34 \\
21 \\
23 \\
13 \\
6 \\
(71)\end{array}$ \\
\hline
\end{tabular}

Les électeurs âgés de moins de 43 ans ont été plutôt déterminés par leur antigaullisme et par les programmes européen et économique du candidat. Les plus de 43 ans ont été plutôt motivés par les qualités personnelles de Jean Lecanuet et par l'idéologie centriste traditionnelle qu'il représentait. Les plus jeunes ont été attirés par des projets politiques précis en opposition avec le système actuel. Les plus âgés se sont montrés moins antigaullistes (sans doute en fonction du rôle historique du général de Gaulle) et moins européens; un passé de guerres et de rivalités vécues avec les pays voisins pourrait expliquer cette réticence.

Cette sensibilité, liée aux événements historiques, . peut expliquer également le vote des électeurs de François Mitterrand :

\begin{tabular}{|c|c|c|}
\hline $\begin{array}{c}\text { Motivations du vote } 1^{\text {ler }} \text { tour } \\
\text { F. MITTERRAND }\end{array}$ & $\begin{array}{l}\text { Moins } \\
\text { de } 43 \text { ans }\end{array}$ & $\begin{array}{l}\text { Plus } \\
\text { de } 43 \text { ans }\end{array}$ \\
\hline $\begin{array}{l}\text { Il est plus proche des ouvriers } \ldots \ldots \ldots \ldots \ldots \\
\text { Contre de Gaulle } \ldots \ldots \ldots \ldots \ldots \ldots \ldots \\
\text { Parce qu'il est jeune } \ldots \ldots \ldots \ldots \ldots \\
\text { Sa politique sociale et économique } \ldots \ldots \ldots \\
\text { Le plus proche de mes idées } \ldots \ldots \ldots \ldots\end{array}$ & $\begin{array}{r}17 \\
21 \\
6 \\
22 \\
17 \\
(143)\end{array}$ & $\begin{array}{r}24 \\
15 \\
10 \\
17 \\
25 \\
(143)\end{array}$ \\
\hline
\end{tabular}

Les électeurs âgés de plus de 43 ans semblent moins antigaullistes que les plus jeunes. D'autre part, leur vote est déterminé par un attachement idéologique et affectif à un système traditionnel : les idées de gauche, la défense de la classe ouvrière. Enfin, ils sont plus sensibles que les plus jeunes à l'âge du candidat. Les moins de 43 ans semblent faire un choix 


\section{Notes de Recherche}

plus politique portant sur des options sociales et économiques précises. On peut faire des constatations analogues à partir des résultats concernant le second tour.

L'âge de l'électeur semble également déterminant dans le cas du vote pour le général de Gaulle. Ce sont les électeurs de plus de 43 ans qui ont été le flus motivés par la «reconnaissance 》, le «nationalisme » et l'admiration pour «le grand homme». Les moins de 43 ans ont voté en fonction de la «stabilité », de la «politique extétieure » et parce que de Gaulle était le seul valable. Les souvenirs semblcnt avoir largement déterminé les premiers, alors que les seconds se sont plus attachés à des problèmes actuels, nécessitant une certaine efficacité :

\begin{tabular}{|c|c|c|}
\hline $\begin{array}{c}\text { Motivations du vote 1er tour } \\
\text { Ga1 DE GAULLE }\end{array}$ & $\begin{array}{l}\text { Moins } \\
\text { de } 43 \text { ans }\end{array}$ & $\begin{array}{l}\text { Plus } \\
\text { de } 43 \text { ans }\end{array}$ \\
\hline 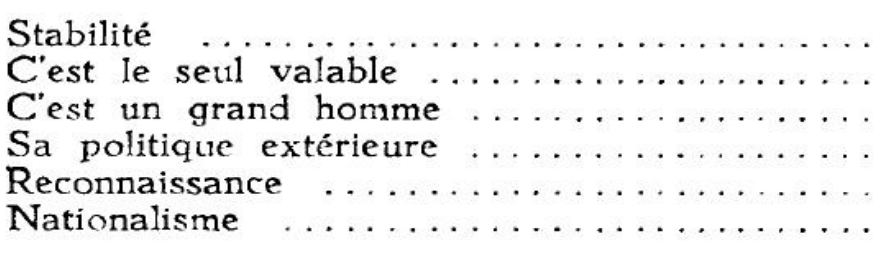 & $\begin{array}{r}40 \\
24 \\
20 \\
18 \\
15 \\
10 \\
(205)\end{array}$ & $\begin{array}{r}30 \\
19 \\
31 \\
12 \\
19 \\
15 \\
(298)\end{array}$ \\
\hline
\end{tabular}

Au second tour, les moins de 43 ans ont également voté en fonction de la «stabilité et de la politique économique du régime actuel ». Ils ont manifesté une forte opposition à Mitterrand. Les plus de 43 ans ont montré à nouveau un certain nationalisme et leur reconnaissance envers le général de Graulle.

Il semblerait que les choix du premier tour aient été plus diversifiés et plus affectifs, les choix du second plus politiques. Il s'agissait de choisir un président de la République, alors qu'au premier tour on pouvait sim. plement exprimer son opinion par son vote.

\begin{tabular}{|c|c|c|}
\hline $\begin{array}{c}\text { Motivations du vote } 2^{\mathrm{e}} \text { tour } \\
\text { G }^{\text {aI }} \text { DE GAULLE }\end{array}$ & $\begin{array}{l}\text { Moins } \\
\text { de } 43 \text { ans }\end{array}$ & $\begin{array}{l}\text { Plus } \\
\text { de } 43 \text { ans }\end{array}$ \\
\hline 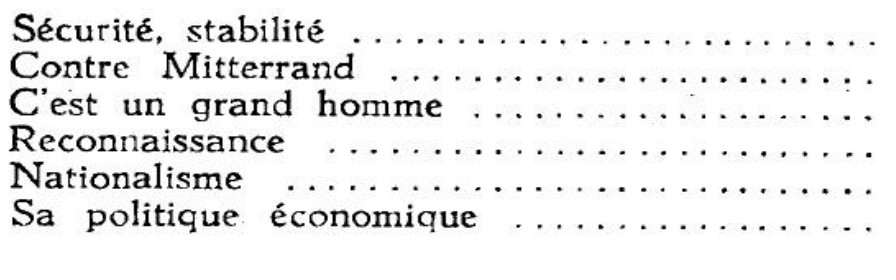 & $\begin{array}{r}27 \\
41 \\
13 \\
8 \\
4 \\
6 \\
(262)\end{array}$ & $\begin{array}{r}23 \\
31 \\
21 \\
11 \\
12 \\
3 \\
(345)\end{array}$ \\
\hline
\end{tabular}




\section{Les Elections Présidentielles}

\section{ZAU D'ETUDES}

\begin{tabular}{|c|c|c|c|}
\hline $\begin{array}{l}\text { ations du vote 1er tour } \\
\mathrm{G}^{\mathrm{at}} \text { DE GAULLE }\end{array}$ & $\begin{array}{l}\text { Pas d'études } \\
\text { et Primaire }\end{array}$ & $\begin{array}{l}\text { Primaire sup. } \\
\text { tech., commerc., } \\
\text { secondaire }\end{array}$ & Supérieur \\
\hline 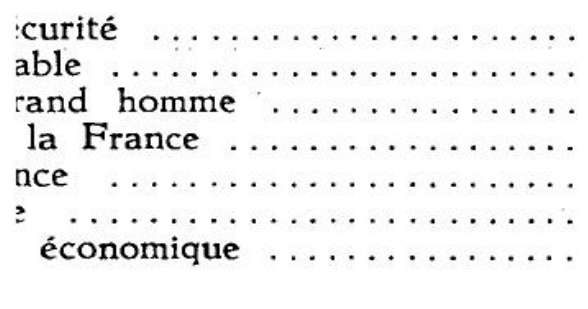 & $\begin{array}{r}32 \\
15 \\
33 \\
8 \\
19 \\
17 \\
3 \\
(187)\end{array}$ & $\begin{array}{c}35 \\
20 \\
27 \\
15 \\
20 \\
10 \\
4 \\
(220)\end{array}$ & $\begin{array}{r}33 \\
35 \\
18 \\
23 \\
9 \\
6 \\
9 \\
(93)\end{array}$ \\
\hline
\end{tabular}

motivations varient avec le niveau d'études, sauf en ce qui concerne ème de la stabilité qui est représenté dans des proportions semblables. plus est, l'importance de la plupart de ces motivations est directement tion du niveau d'études. Les thèmes les plus affectifs vont en dimi$t$ du niveau «études primaires》 au niveau «études supérieures 》, le gaullisme affectif ( (de Gaulle est un grand homme») passe $13 \%$ pour le niveau primaire à $18 \%$ pour les études supérieures, est de même pour la « reconnaissance » et pour le «nationalisme ». :evanche, la proportion des thèmes plus politiques - comparaison yénéral de Gaulle aux autres candidats, ceux-ci étant jugés moins sles - politique extérieure - politique économique - va en augant du niveau primaire au niveau supérieur 14.

peut faire des constatations analogues pour les motivations de vote scond tour :

vations du vote $2^{\circ}$ tour $G^{\star 1}$ DE GAULLE

\section{Pas d'études \\ et Primaire} Primaire sup.
tech., commerc. secondaire

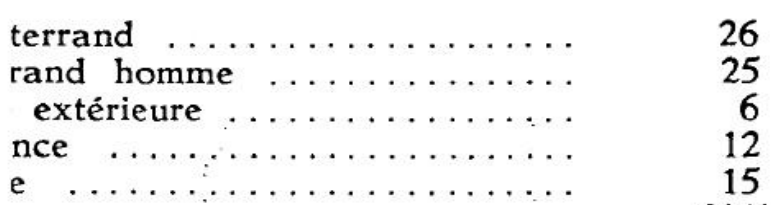

(208)

37
18
9
9
6
$(276)$

(276)
Supérieur

48
8
14
7
5
$(122)$

lans cette analyse des motivations, il faut tenir compte du fait que les nnes de niveau d'études supérieur ont fourni un plus grand nombre iponses que celles de niveau primaire, ce qui explique certains pourcen- Toutefois, il ne semble pas que ce fait puisse modifier la signification :ésultats. 


\section{Notes "de Recherche}

Des différences de motivations suivant les trois niveaux d'étude retenus apparaissent aussi, mais de façon moins nette en ce qui concerne le vote pour François Mitterrand.

Ceux qui ont fait des études primaires ont surtout voté pour lui parce qu'il était "plus proche des ouvriers.», ceux qui ont fait des études supérieures parce qu'il était "le candidat de la gauche», «un démocrate»:

Motivations du vote $1^{\text {er }}$ tour F. MITTERRAND
Pas d'études et Primaire
Primaire sup. tech., commerc. secondaire
Il est plus proche des ouvriers $\ldots \ldots \ldots$

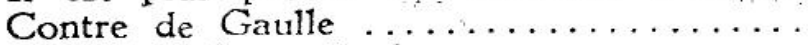

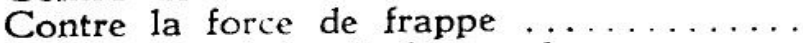

C'est le candidat de la gauche .........

C'est un démocrate $\ldots \ldots \ldots \ldots \ldots \ldots \ldots$

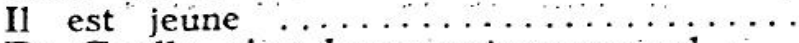

De Gaulle, c'est le pouvoir personnel ....

Sa politique économique et sociale .......

Il est plus proche de mes idées

30
16
5
14
2
7
2
16
18
$(128)$

$\begin{array}{rr}18 & 0 \\ 18 & 23 \\ 13 & 7 \\ 15 & 49 \\ 4 & 12 \\ 8 & 0 \\ 5 & 7 \\ 24 & 18 \\ 19 & 23 \\ (124) & (43)\end{array}$

Le thème «Mitterrand est plus proche des ouvriers 》 correspond à une relation directe entre le candidat et le groupe social auquel appartient l'électeur. alors que. «Mitterrand candidat de la gauche » se réfère plus à une idéologie que l'on partage.

Lopposition ì la force de frappe est fréquemment mentionnée par les électeurs de niveau d'études moyen, mais l'est peu par les électeurs de niveau inférieur et supérieur. Les motivations de vote pour le second tour appellent des remarques analogues à celles que l'on peut faire pour le premicr tour mais certains phénomènes se sont accentués :

\begin{tabular}{|c|c|c|c|c|c|}
\hline $\begin{array}{c}\text { Motivations du vote } 2^{e} \text { tout } \\
\text { F. MITTERRAND }\end{array}$ & $\begin{array}{l}\text { Pas d'études } \\
\text { et Primaire }\end{array}$ & $\begin{array}{l}\text { Primaire s } \\
\text { tech., comm } \\
\text { secondair }\end{array}$ & $\begin{array}{l}\text { sup. } \\
\text { nerc., } \\
\text { ire }\end{array}$ & & Supérieur \\
\hline $\begin{array}{l}\text { Il est plus proche des ouvriers } \ldots \ldots \ldots \ldots \\
\text { Contre de Gaulle } \ldots \ldots \ldots \ldots \\
\text { Pour donner une leçon à de Gaulle } \ldots \ldots \\
\text { Contre la force de frappe } \ldots \ldots \ldots \ldots \\
\text { C'est le candidat de la gauche } \ldots \ldots \ldots \ldots \ldots \\
\text { C'est un démocrate } \ldots \ldots \ldots \ldots \ldots \\
\text { Il est jeune } \ldots \ldots \ldots \ldots \\
\text { De Gaulle, c'est le pouvoir personnel } \ldots \ldots \\
\text { Sa politique économique et sociale } \ldots \ldots \ldots \\
\text { Son programme } \ldots \ldots \ldots \ldots \\
\end{array}$ & $\begin{array}{r}20 \\
18 \\
1 \\
2 \\
11 \\
3 \\
3 \\
5 \\
15 \\
19 \\
(159)\end{array}$ & $\begin{array}{r}10 \\
30 \\
3 \\
9 \\
11 \\
3 \\
8 \\
3 \\
24 \\
16 \\
(138)\end{array}$ & & & $\begin{array}{r}0 \\
49 \\
4 \\
1 \\
24 \\
8 \\
0 \\
6 \\
19 \\
24 \\
(72)\end{array}$ \\
\hline
\end{tabular}




\section{Les Elections · Présidentielles}

Ainsi $49 \%$ des électeurs ayant un niveau d'études supérieur ont voté pour Français Mitterrand par antigaullisme (contre seulement $23 \%$ au premier tour); on constate un phénomène semblable chez les électeurs d'un niveau d'études moyen (l'antigaullisme passe de $18 \%$ au premier tour à $30 \%$ au second tour), alors que cette motivation est représentée de façon à peu près identique au premier et au second tour chez les électeurs de niveau primaire. Peut-être faut-il y voir l'effet du ralliement d'électeurs qui avaient voté pour Lecanuet et Tixier-Vignancour au premier tour et qui n'auraient voté pour «le candidat de tous les républicains » que par hostilité au général de Gaulle. Ce que semblerait confirmer la proportion du thème «c'est le candidat de la gauche » qui diminue quel que soit le niveau d'études, passant en particulier de $49 \%$ à $24 \%$ pour les électeurs de niveau d'étude supérieur. Quant au vote pour Jean Lecanuet, ses motivations sont aussi différenciées suivant le niveau d'études. Les éléments les plus politiques ont motivé en plus forte proportion les électeurs ayant un niveau d'études supétieur, les éléments liés à l'attrait du candidat lui-même ayant été plus importants pour les électeurs de niveau primaire. Ainsi «l'Europe» est mentionnée par $4 \%$ des personnes de niveau primaire, par 30\% de celles qui ont un niveau d'études moyen et par $40 \%$ de celles qui ont fait des études supérieures. D'autre part, la jeunesse du candidat est invoquée par $30 \%$ des électeurs qui n'ont fait que des études primaires et par. $22: \%$ de celles qui ont un niveau d'études supérieures.

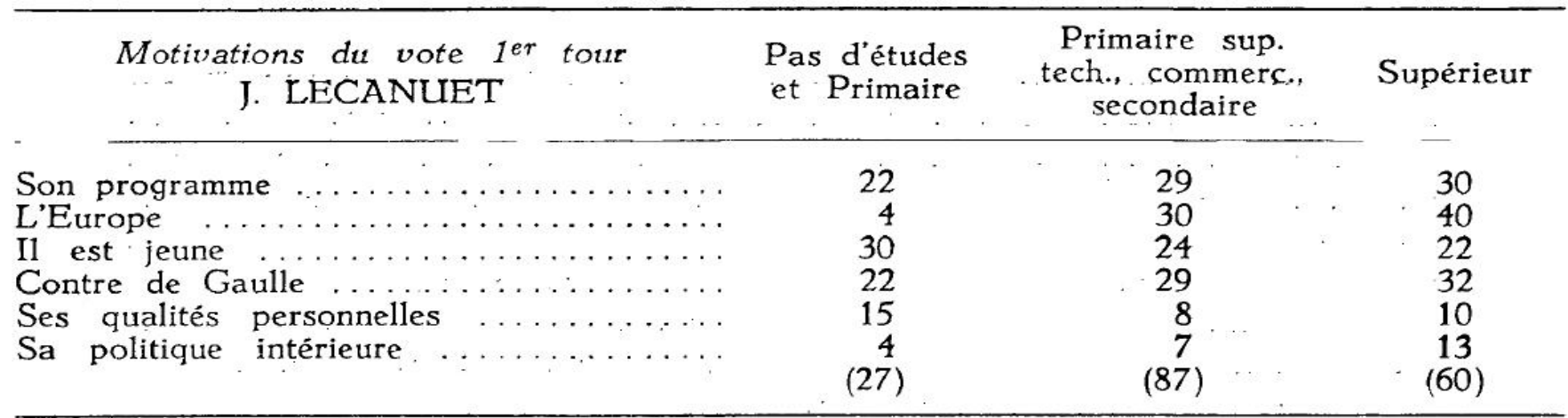

NIVEAU DE REVENUS

\begin{tabular}{|c|c|c|}
\hline Motivations $d u$ vote $1^{\text {er }}$ tour & Moins & Plus \\
\hline$G^{\mathrm{al}}$ DE GAULLE & $1200 \mathrm{~F}$ & $1200 \mathrm{~F}$ \\
\hline 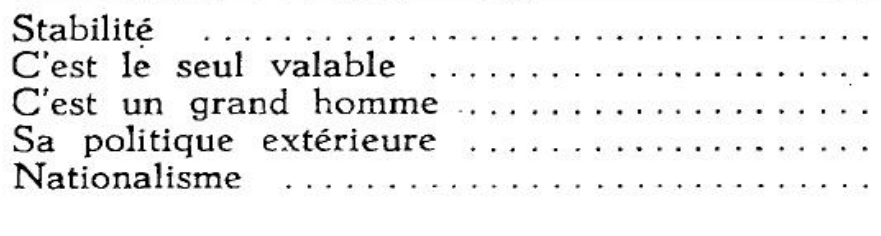 & $\begin{array}{r}30 \\
18 \\
24 \\
9 \\
23 \\
(203)\end{array}$ & $\begin{array}{c}39 \\
24 \\
27 \\
19 \\
8 \\
(270)\end{array}$ \\
\hline
\end{tabular}




\section{Notes de Recherche}

Le nireau de revenus est aussi une variable qui fait apparaître des différences dans les motivations de vote. En ce qui concerne le vote pour le général de Gaulle, l'opposition entre motivations plus politiques (« politique extérieure ») et motivations plus affectives (《nationalisme 》) apparaît clairement : la première de ces motivations est citée par $9 \%$ des électeurs ayant un revenu mensuel inférieur à $1200 \mathrm{~F}$ et par $19 \%$ de ceux qui ont un revenu supérieur; la seconde est mentionnée par 23:\% des électeurs de revenu inférieur et par $81 \%$ des électeurs de revenu supérieur. Les constatations que l'on peut faire sur le second tour sont de nature semblable : $43 \%$ (345) des électeurs de revenu supérieur votent pour le général de Gaulle "pour manifester leur opposition à Mitterrand 》 contre seulement $25 \%$ (226) des électeurs de revenu inférieur :

\begin{tabular}{|c|c|c|}
\hline $\begin{array}{c}\text { Motivations } d u \text { vote } 1^{\text {er }} \text { tour } \\
\text { F. MITTERRAND }\end{array}$ & $\begin{aligned} & \text { Moins } \text { de } \\
& 1200 \mathrm{~F}\end{aligned}$ & $\begin{array}{l}\text { Plus de } \\
1200 \mathrm{~F}\end{array}$ \\
\hline $\begin{array}{l}\text { C'est le candidat de la gauche } \ldots \ldots \ldots \ldots \\
\text { Il est plus proche des ouvriers } \ldots \ldots \ldots \ldots \ldots \\
\text { Contre de Gaulle } \ldots \ldots \ldots \ldots \\
\text { De Gaulle, c'est le pouvoir personnel } \ldots \ldots \\
\text { Sa politique économique et sociale } \ldots \ldots \ldots\end{array}$ & $\begin{array}{r}19 \\
27 \\
10 \\
1 \\
17 \\
(135)\end{array}$ & $\begin{array}{r}22 \\
14 \\
24 \\
7 \\
24 \\
(148)\end{array}$ \\
\hline
\end{tabular}

27:\% des électeurs ayant un revenu mensuel inférieur à $1200 \mathrm{~F}$ ont voté pour François Mitterrand, parce que c'était le candidat «le plus proche des ouvriers : c'est la raison qui détermine le plus grand nombre d'entre eux. En revanche, les électeurs ayant un revenu mensuel supérieur à $1200 \mathrm{~F}$ ont plus voté en fonction de la politique économique et sociale du candidat et de leur antigaullisme ( $c$ contre de Gaulle», " de Gaulle, c'est le pouvoir personnel»). Le vote des premiers traduirait un attachement à une idéologie traditionnelle, le vote des seconds un choix politique et la considération d'objectifs plus précis :

\begin{tabular}{|c|c|c|}
\hline $\begin{array}{c}\text { Motivations } d u \text { vote } 2^{e} \text { tour } \\
\text { F. MITTERRAND }\end{array}$ & $\begin{aligned} \text { Moins de } \\
1200 \mathrm{~F}\end{aligned}$ & $\begin{array}{l}\text { Plus de } \\
1200 \mathrm{~F}\end{array}$ \\
\hline $\begin{array}{l}\text { C'est le candidat de la gauche } \ldots \ldots \ldots \ldots \ldots \\
\text { Il est plus proche des ouvriers } \ldots \ldots \ldots \ldots \\
\text { Contre de Gaulle } \ldots \ldots \ldots \ldots \ldots \\
\text { De Gaulle, c'est le pouvoir personnel } \ldots \ldots \ldots \\
\text { Sa politique sociale et économique } \ldots \ldots \ldots \ldots \\
\text { Son programme } \ldots \ldots \ldots \ldots \ldots \ldots \ldots\end{array}$ & $\begin{array}{r}12 \\
21 \\
18 \\
2 \\
17 \\
22 \\
(147)\end{array}$ & $\begin{array}{r}14 \\
5 \\
36 \\
5 \\
22 \\
16 \\
(202)\end{array}$ \\
\hline
\end{tabular}


La proportion d'électeurs déterminés par leur antigaullisme s'est accentuée du premier au second tour. On peut penser que les divers opposants au gaullisme au premier tour ont reporté leurs suffrages sur le seul adversaire du général de Gaulle au second tour et que ces opposants qui ont voté pour François Mitterrand au second tour seulement appartenaient surtout à des catégories ayant des revenus élevés. Leur choix ne marquerait pas une préférence particulière pour le candidat de la gauche, mais. un moyen de s'opposer effectivement au régime actuel.

\begin{tabular}{|c|c|c|}
\hline $\begin{array}{c}\text { Motivations } d u \text { vote } 1^{\text {er }} \text { tour } \\
\text { J. LECANUET }\end{array}$ & $\begin{array}{l}\text { Moins } \\
1200 \mathrm{~F}\end{array}$ & $\begin{array}{l}\text { Plus } \\
1200 \mathrm{~F}\end{array}$ \\
\hline 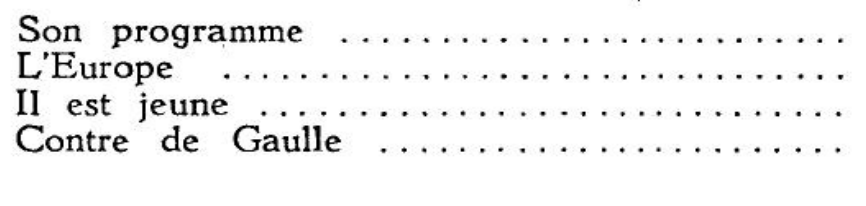 & $\begin{array}{l}35 \\
18 \\
32 \\
21 \\
(34)\end{array}$ & $\begin{array}{c}27 \\
31 \\
21 \\
31 \\
(127)\end{array}$ \\
\hline
\end{tabular}

L'antigaullisme et l'attitude favorable à l'Europe d'une part, le programme du candidat et le fait qu'il est jeune sont les motivations pour lesquelles existent le plus de différences entre électeurs ayant des revenus supérieurs à $1200 \mathrm{~F}$ et ceux qui ont des revenus inférieurs. En particulier le thème de l'Europe est cité par $31 \%$ de ceux qui ont un revenu supérieur et par $18 \%$ seulement de ceux qui ont un revenu inférieur.

\section{PROFESSION DE LA PERSCNNE INTERROGEE}

La faiblesse des effectifs ne permet pas de faire une analyse précise des rapports entre la profession de la personne interrogée et les motivations de son vote. Nous nous bornerons à signaler quelques différences qui semblent significatives.

Dans l'électorat du général de Gaulle, le thème de la «stabilité » est surtout cité par $48 \%$ (48) des patrons de l'industrie et du commerce [34:\% (516) pour l'ensemble] au premier tour; le thème "c'est le seul valable 》 est surtout cité par les professions libérales et cadres supérieurs [32:\% (69) contre $21 \%$ pour l'ensemble], il en est de même pour « la politique extérieure » $(25 \%$ contre $14 \%)$, enfin le thème de «la reconnaissance 》 est surtout cité par les ouvriers [251\% (77) contre $17 \%$ pour l'ensemble]. Au second tour, la catégorie «contre Mitterrand》 remplace «c'est le seul valable», les réponses de ce type atteignent $47 \%$ (90) chez les professions libérales et cadres supérieurs contre $19 \%$ (626) sur l'ensemble. Les autres thèmes se répartissent à peu près comme au premier tour. 


\section{Notes de Recherche}

Parmi les électeurs de François Mitterrand, le thème «c'est le plus proche des ouvriers.» est, bien sûr, évoqué surtout par les ouvriers : $33 \%$ (86) contre $21 \%$ (300) pour l'ensemble; l'opposition au général de. Gaulle se manifeste surtout chcz les professions libérales et cadres supérieurs [36\% (25) contre $18 \%$ sur l'ensemble]; les catégories «contre la force de frappe 》 et "pour sa politique économique et sociale 》 se rencontrent surtout chez les cadres moyens [respectivement $15 \%$ (47) contre 9 \% sur l'ensemble et $32 \%$ contre $21 \% \%]$; " parce que c'est le candidat de la gauche » apparaît dans $36 \%$ (25) des réponses des professions libérales et cadres supérieurs (contre 19\% sur l'ensemble). Au second tour, les fréquences relatives de chaque thème en fonction des professions sont sensiblement les mêmes qu'au premier tour; notons toutéfois que «l'opposition ì de Gaulle » atteint $55 \%$ (38) chez les professions libérales et cadres supérieurs contre $29 \%$ pour l'ensemble.

En reprenant les résultats concernant le niveau d'études et de revenus et la profession de la personne interrogée, on voit que l'antigaullisme a été la motivation spécifique d'électeurs de François Míttetrand, issus d'un groupe de niveau socio-économique et culturel élevé : électeurs ayant fait des études supérieures, ayant un revenu mensuel de plus de $1200 \mathrm{~F}$ et exerçant des professions libérales ou étant des cadres supérieurs. On peut penser que ce groupe a choisi de voter ainsi pour renforcer la position de l'adversaire principal du génćral de Gaulle sans que cela traduise forcément une adhésion complète au programme du «candidat de la gauche ». 